\title{
PLURISUBHARMONIC FUNCTIONS AND THE KÄHLER-RICCI FLOW
}

\author{
By LeI Ni and LuEN-FAi TAM
}

\begin{abstract}
In this paper, a sharp linear trace Li-Yau-Hamilton inequality for Kähler-Ricci flow is proved. The new inequality extends the previous trace Harnack inequality obtained by H.-D. Cao. We also establish sharp gradient estimates for the positive solution of the time-dependent heat equation for some cases. Finally, we apply this new linear trace Li-Yau-Hamilton inequality to study the Liouville properties of the plurisubharmonic functions on complete Kähler manifolds with bounded nonnegative holomorphic bisectional curvature.
\end{abstract}

0. Introduction. Consider the Kähler-Ricci flow on a Kähler manifold $\left(M, g_{\alpha \bar{\beta}}(x)\right)$ :

$$
\frac{\partial}{\partial t} g_{\alpha \bar{\beta}}=-R_{\alpha \bar{\beta}}, \quad g_{\alpha \bar{\beta}}(x, 0)=g_{\alpha \bar{\beta}}(x) .
$$

In this work, $\left(M, g_{\alpha \bar{\beta}}(x)\right)$ will be assumed to be complete and noncompact with bounded nonnegative holomorphic bisectional curvature. Solutions of (0.1) on complete noncompact Kähler manifolds with bounded nonnegative bisectional curvature were extensively studied in a series of paper of Shi [Sh1-3].

Important properties and applications have also been obtained, see [Sh23], [C-Z], [C-T-Z]. In [N-T], the authors studied the Kähler-Ricci flow from another point of view. Namely, solutions of (0.1) are investigated by using the solution to the Poincaré-Lelong equation obtained in [M-S-Y], [N-S-T1]. More precisely, it was proved in [N-S-T1], under some mild average assumptions on scalar curvature $\mathcal{R}_{0}(x)$ of the initial metric $g_{\alpha \bar{\beta}}(x)$, one can solve the PoincaréLelong equation:

$$
\sqrt{-1} \partial \bar{\partial} u_{0}=\operatorname{Ric}_{0}
$$

where $\mathrm{Ric}_{0}$ is the Ricci form of the initial metric. We should mention that (0.2) was solved by Mok-Siu-Yau [M-S-Y] and Mok [M1] in the case when $M$ has maximal volume growth and the scalar curvature has quadratical pointwise decay. Using the solution of $(0.2)$, one can easily find a function $u(x, t)$ so that

Manuscript received October 21, 2001.

Research of the first author supported in part by NSF grant DMS-0196405 and DMS-0203023; research of the second author supported in part by an Earmarked Grant of Hong Kong \#CUHK4217/99P.

American Journal of Mathematics 125 (2003), 0-00. 
$\sqrt{-1} \partial \bar{\partial} u(x, t)=\operatorname{Ric}(x, t)$ where Ric is the Ricci form of the metric $g(t)$. Moreover $u(x, t)$ satisfies the time-dependent heat equation:

$$
\left\{\begin{array}{l}
\left(\frac{\partial}{\partial t}-\Delta\right) u=0 \\
u(x, 0)=u_{0}(x) .
\end{array}\right.
$$

Hence one can study (0.1) by using (0.3). For example, we gave a simple proof of the long time existence of $(0.1)$ under some growth conditions on $\mathcal{R}_{0}$ in [N-T]. Note that in this case $u_{0}$ and $u(\cdot, t)$ are both plurisubharmonic because $g(t)$ has nonnegative holomorphic bisectional curvature [Sh2-3].

This motivates us to study (0.3) for general plurisubharmonic initial data $u_{0}$. Under a rather mild assumption on the growth rate of $u_{0}$, we can prove that $(0.3)$ has a long time solution in the sense that if the Kähler-Ricci flow (0.1) has a solution up to time $T$, then (0.3) also has a unique solution up to time $T$.

The next important question is whether or not under the flow (0.3) the plurisubharmonicity will be preserved. In order to study this problem, we have to study the complex Hessian $u_{\alpha \bar{\beta}}$ of $u$. One can show that $u_{\alpha \bar{\beta}}$ satisfies the complex Lichnerowicz-Laplacian heat equation (see (1.2) for details). We shall prove that if the $\widetilde{\Delta} u_{0}$ is of at most exponential growth, then plurisubharmonicity will be preserved. Here $\widetilde{\Delta}$ is the Laplacian of the initial metric. In fact, we shall prove the result for more general Hermitian symmetric $(1,1)$ tensors which satisfy the Lichnerowicz-Laplacian heat equation. See Proposition 1.1. In case $u_{0}$ is the solution of (0.2), the assumption on the rate of growth of $\widetilde{\Delta} u_{0}$ is the same as the assumption on the rate of growth of the scalar curvature $\mathcal{R}_{0}$, which is assumed to be bounded in [Sh2-3].

There are many important differential Harnack type inequalities for Ricci flow and curvature flows obtained by various people, see [L-Y], [H4], [Cw1-2], [Co12], [A] for examples. Works in this area can be traced back to the fundamental works of Li-Yau [L-Y] and Hamilton [H4]. For this reason, in this paper we shall call this kind of inequalities to be Li-Yau-Hamilton type inequalities, or LYH inequalities for short. In $[\mathrm{C}-\mathrm{H}]$, Chow and Hamilton obtained a linear trace LYH inequality for a symmetric two-tensor on a Riemannian manifold with a family of metric $g(t)$ satisfying the Ricci flow equation so that the initial metric has nonnegative curvature operator. The two-tensor is assumed to satisfy the real Lichnerowicz-Laplacian heat equation. In this paper, using the results of Cao [Co1-2], we shall prove a complex version of Chow-Hamilton's result. More precisely, suppose $(0.1)$ has a solution on $M \times[0, T]$ so that the initial metric has nonnegative bounded holomorphic bisectional curvature. Let $h_{\alpha \bar{\beta}}(x, t)$ be a solution of the complex Lichnerowicz-Laplacian heat equation so that $h_{\alpha \bar{\beta}}(x, 0) \geq$ 0 and $h_{\alpha \bar{\beta}}(x, t)$ satisfies some growth conditions. Then on $M \times(0, T]$, we have

$$
\begin{aligned}
Z= & \frac{1}{2}\left[g^{\alpha \bar{\beta}} \nabla_{\bar{\beta}} \operatorname{div}(h)_{\alpha}+g^{\gamma \bar{\delta}} \nabla_{\gamma} \operatorname{div}(h)_{\bar{\delta}}\right] \\
& +g^{\alpha \bar{\beta}} g^{\gamma \bar{\delta}}\left[R_{\alpha \bar{\delta}} h_{\gamma \bar{\beta}}+\nabla_{\gamma} h_{\alpha \bar{\delta}} V_{\bar{\beta}}+\nabla_{\bar{\beta}} h_{\alpha \bar{\delta}} V_{\gamma}+h_{\alpha \bar{\delta}} V_{\bar{\beta}} V_{\gamma}\right]+\frac{H}{t} \geq 0
\end{aligned}
$$


where $\operatorname{div}(h)_{\alpha}=g^{\gamma \bar{\delta}} \nabla_{\gamma} h_{\alpha \bar{\delta}}, \operatorname{div}(h)_{\bar{\beta}}=g^{\gamma \bar{\delta}} \nabla_{\bar{\delta}} h_{\gamma \bar{\beta}}, H$ is the trace of $h_{\alpha \bar{\beta}}$ with respect to $g_{\alpha \bar{\beta}}(x, t)$, and $V$ is any vector field of type $(1,0)$. See Theorem 1.1 for details. In case $h_{\alpha \bar{\beta}}$ is the complex Hessian of a plurisubharmonic solution of (0.3) so that $u_{0}$ is not harmonic, then our result implies that $w=u_{t}$ satisfies

$$
w_{t}-\frac{|\nabla w|^{2}}{w}+\frac{w}{t} \geq 0
$$

which extends Cao's trace LYH type inequality for the scalar curvature [Co1-2]. Unlike [C-H], which mainly considers compact manifolds and is not very specific on noncompact manifolds, we need the growth conditions on $h_{\alpha \bar{\beta}}$ so that one can apply the maximum principle in $[\mathrm{N}-\mathrm{T}]$.

As an application of the above results on the study of (0.3) and the linear trace LYH type inequality, we shall study Liouville properties for plurisubharmonic functions on $\left(M, g_{\alpha \bar{\beta}}(x)\right)$. Suppose $(0.1)$ has long time solution. Then we have the following:

THEOREM 3.1. Let $\left(M, g_{\alpha \bar{\beta}}(x)\right)$ be a complete noncompact manifold with bounded nonnegative holomorphic bisectional curvature so that the (0.1) has long time solution. Suppose $u_{0}$ is a plurisubharmonic function such that (i) $u$ is bounded; and (ii) $\widetilde{\Delta} u_{0}(x) \leq \exp \left(a\left(1+r_{0}(x)\right)\right.$ for some constant $a>0$. Then $u_{0}$ must be constant.

In $\mathbb{C}^{m}$, a plurisubharmonic function with sub-logarithmic growth must be constant. It is conjectured that this is still true for complete noncompact Kähler manifolds with nonnegative Ricci curvature. In this paper, we shall also prove that in some cases, the condition that $u_{0}$ is bounded in the above theorem can be relaxed. For example, one can prove that if the scalar curvature has quadratic decay in the average sense, then Theorem 3.1 is still true if the condition (i) is replaced by the condition that $u_{0}$ has sub-logarithmic growth. This is a special case of a more general result, see Theorem 3.2. In particular, when $u_{0}(x)$ is the solution of (0.2), the Liouville result mentioned above implies the gap theorem proved in $[\mathrm{C}-\mathrm{Z}]$ and $[\mathrm{N}-\mathrm{T}]$. For previous results of Liouville properties of plurisubharmonic functions, please see $[\mathrm{N}]$, [N-S-T1-2], [N-T].

As a by-product of our argument, we also prove a Li-Yau type differential inequality for the positive plurisubharmonic solution $u(x, t)$ of $(0.3)$ (see Theorem 2.2). Namely, we have

$$
\frac{u_{t}}{u}-\frac{|\nabla u|^{2}}{u^{2}}+\frac{m}{t} \geq 0
$$

exactly as in [L-Y] for a fixed metric. Hopefully, this differential inequality will have applications to the study of the plurisubharmonic functions, the Kähler-Ricci flow and other problems.

Here is the organization of the paper. In $\S 1$, we shall prove the preservation of nonnegativity of $(1,1)$ tensors and the linear trace LYH type inequality for 
$(1,1)$ tensors. In $\S 2$, we shall study the initial value problem $(0.3)$ and prove the Li-Yau type inequality for the positive solution to (0.3). In $\S 3$, we shall study Liouville properties of plurisubharmonic functions.

Acknowledgments. The authors would like to thank Ben Chow for his interest and discussions and also Huai-Dong Cao for discussions.

1. A Li-Yau-Hamilton inequality. In this section we shall prove a linear trace Li-Yau-Hamilton inequality which is the Kähler version of the one obtained by Chow and Hamilton in [C-H]. Just as Chow-Hamilton's LYH inequality extends the trace Li-Yau-Hamilton inequality of Hamilton [H4] our differential inequality extends the trace LYH inequality of Cao [Co1] for the scalar curvature. Applications of this new inequality will be given in the following sections.

Let $\left(M^{m}, g_{\alpha \bar{\beta}}(x)\right)$ be a complete noncompact Kähler manifold with bounded nonnegative holomorphic bisectional curvature. Because of the results in [Sh1], in this section we always assume that solution of the following Ricci-Kähler flow exists on $M \times[0, T]$

$$
\left\{\begin{array}{l}
\frac{\partial}{\partial t} g_{\alpha \bar{\beta}}=-R_{\alpha \bar{\beta}} \\
g_{\alpha \bar{\beta}}(x, 0)=g_{\alpha \bar{\beta}}(x)
\end{array}\right.
$$

such that on $M \times[0, T]$,

(i) $g_{\alpha \bar{\beta}}(x, t)$ is nonincreasing in $t$ and is uniformly equivalent to $g_{\alpha \bar{\beta}}(x, 0)$;

(ii) the curvature tensors of $g_{\alpha \bar{\beta}}(x, t)$ are uniformly bounded;

(iii) there exists a constant $C$ such that

$$
|\nabla \operatorname{Rm}|(x, t) \leq \frac{C}{t^{\frac{1}{2}}} ; \text { and }
$$

(iv) $g_{\alpha \bar{\beta}}(x, t)$ has nonnegative holomorphic bisectional curvature.

Sufficient conditions that (1.1) has long time existence are given in [Sh2-3], [N-T], see also [C-T-Z] for the surfaces case.

In this work, we will use the maximum principle of the authors [N-T, Theorem 1.2] from time to time. For the convenience of the readers, we include the statement of this maximum principle here.

THEOREM 1.1. Let $g_{i j}(x, t)$ be a smooth family of complete Riemannian metrics defined on $M$ with $0 \leq t \leq T$ for some $T>0$ such that for any $T \geq t_{2} \geq t_{1} \geq 0$

$$
C g_{i j}\left(x, t_{1}\right) \leq g_{i j}\left(x, t_{2}\right) \leq g_{i j}\left(x, t_{1}\right)
$$

for some constant $C>0$ for all $x \in M$ and let $f(x, t)$ be a smooth function such 
that $\left(\Delta-\frac{\partial}{\partial t}\right) f(x, t) \geq 0$ whenever $f(x, t) \geq 0$. Assume that

$$
\int_{0}^{T} \int_{M} \exp \left(-a r_{0}^{2}(x)\right) f_{+}^{2}(x, s) d V_{0} d s<\infty
$$

for some $a>0$, where $r_{0}(x)$ is the distance function to a fixed point $o \in M$ with respect to $g_{i j}(x, 0)$. Suppose $f(x, 0) \leq 0$ for all $x \in M$. Then $f(x, t) \leq 0$ for all $(x, t) \in M \times[0, T]$.

In the following let $h_{\alpha \bar{\beta}}(x, t)$ be a Hermitian symmetric tensor defined on $M \times[0, T]$, which is also deformed by the complex Lichnerowicz-Laplacian heat equation:

$$
\left(\frac{\partial}{\partial t}-\Delta\right) h_{\gamma \bar{\delta}}=R_{\beta \bar{\alpha} \gamma \bar{\delta}} h_{\alpha \bar{\beta}}-\frac{1}{2}\left(R_{\gamma \bar{p}} h_{p \bar{\delta}}+R_{p \bar{\delta}} h_{\gamma \bar{p}}\right)
$$

We shall obtain a LYH inequality for $h_{\alpha \bar{\beta}}$ provided $h_{\alpha \bar{\beta}}$ is nonnegative and does not grow very fast on $M \times[0, T]$. In application, usually we only know that $h_{\alpha \bar{\beta}}$ is nonnegative initially. Hence we shall discuss conditions on $h_{\alpha \bar{\beta}}$ so that nonnegativity is preserved under the flow. The following lemma is basically from [H4, Lemma 5.1].

Lemma 1.1. For any $a>0$ and $C>0$ there exists a positive function $\phi(x, t)$ and $b>0$ such that $\exp \left(b\left(r_{0}(x)+1\right)\right) \geq \phi(x, t) \geq \exp \left(a\left(r_{0}(x)+1\right)\right)$ and

$$
\left(\frac{\partial}{\partial t}-\Delta\right) \phi \geq C \phi
$$

on $M \times[0, T]$, where $r_{0}(x)$ is the distance from a fixed point $o$ with respect to the initial metric $g(0)$.

Proof. By Lemma 5.1 in [H4], there is a smooth function $f(x)$ and a constant $C_{1}>0$ on $M$ such that

$$
\begin{gathered}
C_{1}^{-1}\left(1+r_{0}(x)\right) \leq f(x) \leq C_{1}\left(1+r_{0}(x)\right) \\
|\nabla f|+\left|\nabla^{2} f\right| \leq C_{1}
\end{gathered}
$$

As in [H4], we can choose $\phi(x, t)=\exp (A t+\alpha f(x))$ for suitable positive constants $A$ and $\alpha$, then $\phi$ will be the required function.

To simplify notations, in the rest of the section, let $\|h\|$ be the norm of $h$ with respect to $g_{\alpha \bar{\beta}}(x, t)$,

$$
\left\{\begin{array}{l}
\Phi=\|h\|^{2} \\
\Psi=\|\nabla h\|^{2}=\sum_{\alpha \beta \gamma}\left(\left\|\nabla_{\gamma} h_{\alpha \bar{\beta}}\right\|^{2}+\left\|\nabla_{\bar{\gamma}} h_{\alpha \bar{\beta}}\right\|^{2}\right) \\
\Lambda=\|\nabla \nabla h\|^{2}=\sum_{\alpha \beta \gamma \delta}\left(\left\|\nabla_{\delta} \nabla_{\gamma} h_{\alpha \bar{\beta}}\right\|^{2}+\left\|\nabla_{\delta} \nabla_{\bar{\gamma}} h_{\alpha \bar{\beta}}\right\|^{2}\right) .
\end{array}\right.
$$


Suppose $h$ satisfies (1.2), then direct computations show (see [H1] for example):

$$
\begin{aligned}
& \left(\frac{\partial}{\partial t}-\Delta\right) \Phi=-\Psi+A, \\
& \left(\frac{\partial}{\partial t}-\Delta\right) \Psi=-\Lambda+B,
\end{aligned}
$$

where $A$ and $B$ satisfy the following conditions: There exists a constant $C>0$ such that $|A| \leq C \Phi$ and $t|B| \leq C(\Phi+\Psi)$ on $M \times[0, T]$. Here we have used properties (ii) and (iii) of $g_{\alpha \bar{\beta}}$.

Moreover, in normal coordinates

$$
\begin{aligned}
\|\nabla \Phi\|^{2} & =\sum_{\alpha} \Phi_{\alpha} \Phi_{\bar{\alpha}} \\
& =\sum_{\alpha}\left(\sum_{\xi, \tau} h_{\xi \bar{\tau}, \alpha} h_{\bar{\xi} \tau}+h_{\xi \bar{\tau}} h_{\bar{\xi} \tau, \alpha}\right)\left(\sum_{\xi, \tau} h_{\xi \bar{\tau}, \bar{\alpha}} h_{\bar{\xi} \tau}+h_{\xi \bar{\tau}} h_{\bar{\xi} \tau, \bar{\alpha}}\right) \\
& \leq 4\|h\|^{2} \sum_{\alpha}\left[\left(\sum_{\xi, \tau}\left|h_{\xi \bar{\tau}, \alpha}\right|^{2}\right)^{\frac{1}{2}}\left(\sum_{\xi, \tau}\left|h_{\xi \bar{\tau}, \bar{\alpha}}\right|^{2}\right)^{\frac{1}{2}}\right] \\
& \leq 2\|h\|^{2} \sum_{\alpha, \xi \tau}\left(\left|h_{\xi \bar{\tau}, \alpha}\right|^{2}+\left|h_{\xi \bar{\tau}, \bar{\alpha}}\right|^{2}\right) .
\end{aligned}
$$

Hence

$$
\|\nabla \Phi\|^{2} \leq 2 \Phi \Psi
$$

Similarly,

$$
\|\nabla \Psi\|^{2} \leq 2 \Psi \Lambda
$$

LEMMA 1.2. Let $h_{\alpha \bar{\beta}}$ be a tensor satisfying (1.2). Suppose

$$
\left\|h_{\alpha \bar{\beta}}(x, 0)\right\| \leq \exp \left(a\left(1+r_{0}(x)\right)\right.
$$

and

$$
\int_{0}^{T} \int_{M} \exp \left(-b r_{0}^{2}(x)\right)\|h\|^{2}(x, t) d V_{t} d t<\infty
$$

for some positive constants $a$ and $b$. Then there exists a positive constant $c>0$ such that

$$
\left\|h_{\alpha \bar{\beta}}(x, t)\right\| \leq \exp \left(c\left(1+r_{0}(x)\right)\right.
$$

on $M \times[0, T]$. 
Proof. By (1.4) and (1.8), it is easy to see that

$$
\left(\frac{\partial}{\partial t}-\Delta\right)\left[e^{-C_{1} t}(1+\Phi)^{\frac{1}{2}}\right] \leq 0
$$

for some constant $C_{1}>0$.

By Lemma 1.1, there exists a function $\phi(x, t)$ and constant $c>0$ such that $\exp \left(c\left(r_{0}(x)+1\right)\right) \geq \phi(x, t) \geq \exp \left(a\left(r_{0}(x)+1\right)\right)$ and

$$
\left(\frac{\partial}{\partial t}-\Delta\right) \phi \geq C \phi
$$

with $C>0$. By (1.9), (1.10) and (1.11), we have $\phi+1 \geq(1+\Phi)^{\frac{1}{2}}$ by the maximum principle Theorem 1.1. The lemma follows by choice of an even larger $c$.

Next we shall prove that nonnegativity of $h$ will be preserved by the flow under certain conditions.

Proposition 1.1. Suppose $h_{\alpha \bar{\beta}}$ satisfy (1.2) and the conditions (1.8) and (1.9) of Lemma 1.2. Suppose also that $h_{\alpha \bar{\beta}}(x, 0) \geq 0$. Then $h_{\alpha \bar{\beta}}(x, t) \geq 0$ for $t>0$.

Proof. By Lemma 1.2, there exists a constant $c>0$ such that

$$
\|h\|(x, t) \leq \exp \left(c\left(1+r_{0}(x)\right)\right) .
$$

By Lemma 1.1, for any $C^{\prime}>0$, there exists a function $\phi$ such that

$$
\exp \left(c^{\prime}\left(1+r_{0}(x)\right)\right) \geq \phi \geq \exp \left(2 c\left(1+r_{0}(x)\right)\right)
$$

and

$$
\left(\frac{\partial}{\partial t}-\Delta\right) \phi>C^{\prime} \phi
$$

It is enough to show that $h_{\alpha \bar{\beta}}(x, t)+\epsilon \phi g_{\alpha \bar{\beta}}(x, t)>0$, for any $\epsilon>0$. Now we calculate

$$
\begin{aligned}
\left(\frac{\partial}{\partial t}-\Delta\right)\left(h_{\alpha \bar{\beta}}+\epsilon \phi g_{\alpha \bar{\beta}}\right)= & R_{\alpha \bar{\beta} \gamma \bar{\delta}}\left(h_{\bar{\gamma} \delta}+\epsilon \phi g_{\bar{\gamma} \delta}\right)-\frac{1}{2} R_{\alpha \bar{p}}\left(h_{p \bar{\beta}}+\epsilon \phi g_{p \bar{\beta}}\right) \\
& -\frac{1}{2} R_{p \bar{\beta}}\left(h_{\alpha \bar{p}}+\epsilon \phi g_{\alpha \bar{p}}\right) \\
& +\epsilon\left(\phi_{t}-\Delta \phi\right) g_{\alpha \bar{\beta}}-\epsilon \phi R_{\alpha \bar{\beta}} .
\end{aligned}
$$

Here we have used (1.2) and the Ricci flow equation. By (1.12), (1.13) and the fact that at $t=0, h_{\alpha \bar{\beta}}+\epsilon \phi g_{\alpha \bar{\beta}}>0$, if $h_{\alpha \bar{\beta}}(x, t)+\epsilon \phi g_{\alpha \bar{\beta}}(x, t)>0$ fails to hold at some $t>0$, then there is $\left(x_{0}, t_{0}\right)$ and unit $(1,0)$ vector at $x_{0}$ with $t_{0}>0$ such 
that $\left(h_{\alpha \bar{\beta}}\left(x_{0}, t_{0}\right)+\epsilon \phi g_{\alpha \bar{\beta}}(x, t)\right) v^{\alpha} \bar{v}^{\beta}=0$ and $t_{0}$ is the first time that happens. As in [Cw3], we can extend $v$ in a neighborhood in space-time of $\left(x_{0}, t_{0}\right)$ such that $\nabla v$ and $\Delta v=0$ at $\left(x_{0}, t_{0}\right)$ with respect to the metric $g\left(t_{0}\right)$ and such that $v$ is independent of time. Hence at $\left(x_{0}, t_{0}\right)$ we have

$$
\begin{aligned}
0 \geq & \left(\frac{\partial}{\partial t}-\Delta\right)\left[\left(h_{\alpha \bar{\beta}}+\epsilon \phi g_{\alpha \bar{\beta}}\right) v^{\alpha} \bar{v}^{\beta}\right] \\
= & {\left[\left(\frac{\partial}{\partial t}-\Delta\right)\left(h_{\alpha \bar{\beta}}+\epsilon \phi g_{\alpha \bar{\beta}}\right)\right] v^{\alpha} \bar{v}^{\beta} } \\
= & R_{\alpha \bar{\beta} \gamma \bar{\delta}}\left(h_{\bar{\gamma} \delta}+\epsilon \phi g_{\bar{\gamma} \delta}\right) v^{\alpha} \bar{v}^{\beta}-\frac{1}{2} R_{\alpha \bar{p}}\left(h_{p \bar{\beta}}+\epsilon \phi g_{p \bar{\beta}}\right) v^{\alpha} \bar{v}^{\beta} \\
& -\frac{1}{2} R_{p \bar{\beta}}\left(h_{\alpha \bar{p}}+\epsilon \phi g_{\alpha \bar{p}}\right) v^{\alpha} \bar{v}^{\beta}+\epsilon\left(\phi_{t}-\Delta \phi\right) g_{\alpha \bar{\beta}} v^{\alpha} \bar{v}^{\beta}-\epsilon \phi R_{\alpha \bar{\beta}} v^{\alpha} \bar{v}^{\beta} .
\end{aligned}
$$

Since $v$ minimizes $h_{\alpha \bar{\beta}}+\epsilon \phi g_{\alpha \bar{\beta}}$ among all $(1,0)$ unit vectors at $x_{0}$, first variation gives

$$
\left(h_{\alpha \bar{\beta}}+\epsilon \phi g_{\alpha \bar{\beta}}\right) v^{\alpha}=\left(h_{\alpha \bar{\beta}}+\epsilon \phi g_{\alpha \bar{\beta}}\right) \bar{v}^{\beta}=0 .
$$

Using also the fact that $M, g\left(t_{0}\right)$ has nonnegative holomorphic bisectional curvature, we conclude that

$$
0 \geq \epsilon\left(\phi_{t}-\Delta \phi\right)-\epsilon \phi R_{\alpha \bar{\beta}} v^{a} \bar{v}^{\beta}>0
$$

for sufficiently large $C^{\prime}$, since $|R m|$ is bounded. This is a contradiction.

We should remark that the result is still true if $M$ is compact. In this case, there is no need to impose a growth condition on $h_{\alpha \bar{\beta}}$. Moreover if $h_{\alpha \bar{\beta}}(x, 0)$ is positive at some point, then $h_{\alpha \bar{\beta}}(x, t)$ will be positive for all $t>0$.

In order to apply the maximum principle we also need the following estimates.

Lemma 1.3. Let $h_{\alpha \bar{\beta}}$ as in Lemma 1.2. Then for any $a>0$,

$$
\begin{aligned}
& \int_{0}^{T} \int_{M} e^{-a r_{0}^{2}(x)} \Psi(x, t) d V_{t} d t<\infty, \\
& \int_{0}^{T} \int_{M} t e^{-a r_{0}^{2}(x)} \Lambda(x, t) d V_{t} d t<\infty
\end{aligned}
$$

and

$$
\int_{0}^{T} \int_{M} t e^{-a r_{0}^{2}(x)} \Psi^{2}(x, t) d V_{t} d t<\infty
$$

Proof. Let $f(x)$ be a smooth function such that $0 \leq f \leq 1, f=1$ on $B_{0}(o, R)$, $f=0$ outside $B_{0}(o, 2 R)$ and $|\widetilde{\nabla} f| \leq C / R$ for some constant $C$ independent of $R$. 
Here $B_{0}(o, R)$ is the geodesic ball with center at $o$ and radius $R$ with respect to $g(0)$. Multiply (1.4) by $f^{2}$ and integrating by parts, we have:

$$
\begin{aligned}
\int_{0}^{T} \int_{M} f^{2} \Psi d V_{t} d t \leq & -\int_{0}^{T} \int_{M} f^{2}\left(\frac{\partial}{\partial t}-\Delta\right) \Phi d V_{t} d t+C_{1} \int_{0}^{T} \int_{M} f^{2} \Phi d V_{t} d t \\
\leq & \int_{M} f^{2} \Phi d V_{0} d t+2 \int_{0}^{T} \int_{M} f|\nabla f||\nabla \Phi| d V_{t} d t \\
& +C_{1} \int_{0}^{T} \int_{M} f^{2} \Phi d V_{t} d t
\end{aligned}
$$

for some constant $C_{1}$. Here we have used the fact that $d V_{t}$ is nonincreasing. Using (1.6) and Schwarz inequality, we have

$$
\int_{0}^{T} \int_{M} f^{2} \Psi d V_{t} d t \leq C_{2}\left[\int_{M} f^{2} \Phi d V_{0} d t+\int_{0}^{T} \int_{M}\left(f^{2}+|\widetilde{\nabla} f|^{2}\right) \Phi d V_{0} d t\right],
$$

for some constant $C_{2}$, where we have used the fact that $g(t)$ and $g(0)$ are equivalent in $[0, T]$. Hence

$$
\int_{0}^{T} \int_{B_{0}(o, R)} \Psi d V_{t} d t \leq C_{3}\left[\int_{B_{0}(o, 2 R)} \Phi d V_{0} d t+\int_{0}^{T} \int_{B_{0}(o, 2 R)} \Phi d V_{0} d t\right],
$$

for some constant $C_{3}$, where we assume $R \geq 1$. By Lemma 1.1, $\|h\|$ is at most of exponential growth, hence it is easy to see (1.16) is true because $g(0)$ has nonnegative Ricci curvature.

To prove (1.17), multiplying (1.5) by $t f^{2}$ and integrating by parts we have for $R \geq 1$,

$$
\begin{aligned}
\int_{0}^{T} \int_{M} t f^{2} \Lambda d V_{t} d t \\
\leq-\int_{0}^{T} \int_{M} t f^{2}\left(\frac{\partial}{\partial t}-\Delta\right) \Psi d V_{t} d t+C_{4} \int_{0}^{T} \int_{M} f^{2}(\Phi+\Psi) d V_{t} d t \\
\leq \int_{0}^{T} \int_{M} f^{2} \Psi d V_{t} d t+2 \int_{0}^{T} \int_{M} t f|\nabla f||\nabla \Psi| d V_{t} d t \\
\quad+C_{4} \int_{0}^{T} \int_{M} f^{2}(\Phi+\Psi) d V_{t} d t \\
\leq C_{5} \int_{0}^{T} \int_{M}\left(f^{2}+|\widetilde{\nabla} f|^{2}\right)(\Phi+\Psi) d V_{t} d t+\frac{1}{2} \int_{0}^{T} \int_{M} t f^{2} \Lambda d V_{t} d t
\end{aligned}
$$

for some constants $C_{4}, C_{5}$, where we have used (1.7). Hence if $R$ is large

$$
\int_{0}^{T} \int_{B_{0}(o, R)} t \Lambda d V_{t} d t \leq 3 C_{5} \int_{0}^{T} \int_{B_{0}(o, 2 R)}(\Phi+\Psi) d V_{t} d t
$$

Combining (1.19) and (1.20), we can conclude that (1.17) is true. 
To prove (1.18), multiplying (1.4) by $t f^{2} \Psi$ and integrating by parts, we have

$$
\begin{aligned}
& \int_{0}^{T} \int_{M} t f^{2} \Psi^{2} d V_{t} d t \\
& \leq-\int_{0}^{T} \int_{M} t f^{2} \Psi\left(\frac{\partial}{\partial t}-\Delta\right) \Phi d V_{t} d t+\int_{0}^{T} \int_{M} t f^{2} \Psi A d V_{t} d t \\
& \leq C_{6} \int_{0}^{T} \int_{M} f^{2} \Psi \Phi d V_{t} d t+\int_{0}^{T} \int_{M} t f^{2} t\left(\Phi \frac{\partial}{\partial t} \Psi+\Psi \Delta \Phi\right) d V_{t} d t \\
& \leq C_{6} \int_{0}^{T} \int_{M} f^{2} \Psi \Phi d V_{t} d t+\int_{0}^{T} \int_{M} t f^{2}(\Phi \Delta \Psi+\Psi \Delta \Phi) d V_{t} d t \\
&+\int_{0}^{T} \int_{M} t f^{2} \Phi B d V_{t} d t \\
& \leq C_{7} \int_{0}^{T} \int_{M} f^{2}(\Psi+\Phi) \Phi d V_{t} d t+2 \int_{0}^{T} \int_{M} t f^{2}|\nabla \Phi||\nabla \Psi| d V_{t} d t \\
&+2 \int_{0}^{T} \int_{M} t f|\nabla f|(\Phi|\nabla \Psi|+\Psi|\nabla \Phi|) d V_{t} d t
\end{aligned}
$$

for some constants $C_{6}, C_{7}$, where we have used (1.5). Apply (1.6) and (1.7) to $|\nabla \Phi|$ and $|\Psi|$ respectively, and use Schwarz inequality, we have,

$$
\int_{0}^{T} \int_{M} t f^{2} \Psi^{2} d V_{t} d t \leq C_{8}\left(\int_{0}^{T} \int_{M}\left(f^{2}+|\widetilde{\nabla} f|^{2}\right)(\Phi+\Psi) \Phi d V_{t} d t+t f^{2} \Lambda \Phi d V_{t} d t\right)
$$

for some constant $C_{8}$. Combining this with (1.16) and (1.17) and the fact that $\Phi$ grows at most exponentially, we can conclude that (1.18) is true.

Remark 1.1. (1.17) and (1.18) imply that for any $\epsilon>0$,

$$
\int_{\epsilon}^{T} \int_{M} t e^{-a r_{0}^{2}(x)} \Lambda(x, t) d V_{t} d t<\infty
$$

and

$$
\int_{\epsilon}^{T} \int_{M} t e^{-a r_{0}^{2}(x)} \Psi^{2}(x, t) d V_{t} d t<\infty
$$

Now we are ready to prove a LYH inequality. Let $\operatorname{div}(h)_{\alpha}=g^{\gamma \bar{\delta}} \nabla_{\gamma} h_{\alpha \bar{\delta}}$ and $\operatorname{div}(h)_{\bar{\beta}}=g^{\gamma \bar{\delta}} \nabla_{\bar{\delta}} h_{\gamma \bar{\beta}}$. Consider the quantity

$$
Z=g^{\alpha \bar{\beta}} g^{\gamma \bar{\delta}}\left[\frac{1}{2}\left(\nabla_{\bar{\beta}} \nabla_{\gamma}+\nabla_{\gamma} \nabla_{\bar{\beta}}\right) h_{\alpha \bar{\delta}}+R_{\alpha \bar{\delta}} h_{\gamma \bar{\beta}}\right.
$$




$$
\begin{gathered}
\left.+\left(\nabla_{\gamma} h_{\alpha \bar{\delta}} V_{\bar{\beta}}+\nabla_{\bar{\beta}} h_{\alpha \bar{\delta}} V_{\gamma}\right)+h_{\alpha \bar{\delta}} V_{\bar{\beta}} V_{\gamma}\right]+\frac{H}{t} \\
=\frac{1}{2}\left[g^{\alpha \bar{\beta}} \nabla_{\bar{\beta}} \operatorname{div}(h)_{\alpha}+g^{\gamma \bar{\delta}} \nabla_{\gamma} \operatorname{div}(h)_{\bar{\delta}}\right] \\
+g^{\alpha \bar{\beta}} g^{\gamma \bar{\delta}}\left[R_{\alpha \bar{\delta}} h_{\gamma \bar{\beta}}+\nabla_{\gamma} h_{\alpha \bar{\delta}} V_{\bar{\beta}}+\nabla_{\bar{\beta}} h_{\alpha \bar{\delta}} V_{\gamma}+h_{\alpha \bar{\delta}} V_{\bar{\beta}} V_{\gamma}\right]+\frac{H}{t},
\end{gathered}
$$

where $H$ is the trace of $h_{\alpha \bar{\beta}}$ with respect to $g_{\alpha \bar{\beta}}(x, t)$.

THEOREM 1.2. Let $h_{\alpha \bar{\beta}}$ be a Hermitian symmetric tensor satisfying (1.2) on $M \times[0, T]$. Suppose $h_{\alpha \bar{\beta}}(x, 0) \geq 0$ and satisfies (1.8) and (1.9) in Lemma 1.2. Then $Z \geq 0$ on $M \times(0, T]$ for any smooth vector field $V$ of type $(1,0)$.

In order to prove the theorem, we need to compute $\left(\frac{\partial}{\partial t}-\Delta\right) Z$. As in $[\mathrm{C}-\mathrm{H}]$, we need to calculate $\left(\frac{\partial}{\partial t}-\Delta\right) Z$. We break up the computations into several lemmas.

LEMMA 1.4. Under normal coordinates at a point,

$$
\left(\frac{\partial}{\partial t}-\Delta\right)\left(\operatorname{div}(h)_{\alpha}\right)=R_{s \bar{t}} \nabla_{t} h_{\alpha \bar{s}}+\nabla_{\alpha} R_{s \bar{t}} h_{\bar{s} t}-\frac{1}{2} R_{\alpha \bar{t}}\left(\operatorname{div}(h)_{t}\right) .
$$

Proof. Direct calculation shows

$$
\begin{aligned}
\frac{\partial}{\partial t}\left(g^{\gamma \bar{\delta}} \nabla_{\gamma} h_{\alpha \bar{\delta}}\right)= & \frac{\partial}{\partial t}\left[g^{\gamma \bar{\delta}}\left(\partial_{\gamma} h_{\alpha \bar{\delta}}-\Gamma_{\alpha \gamma}^{p} h_{p \bar{\delta}}\right)\right] \\
= & g^{\gamma \bar{t}} R_{s \bar{t}} g^{s \bar{\delta}} \nabla_{\gamma} h_{\alpha \bar{\delta}}+g^{\gamma \bar{\delta}} \nabla_{\gamma}\left(\frac{\partial}{\partial t} h_{\alpha \bar{\delta}}\right)-g^{\gamma \bar{\delta}}\left(\frac{\partial}{\partial t} \Gamma_{\alpha \gamma}^{p}\right) h_{p \bar{\delta}} \\
= & R_{s \bar{t}} \nabla_{t} h_{\alpha \bar{s}}+\nabla_{\gamma}\left(\Delta h_{\alpha \bar{\gamma}}+R_{\alpha \bar{\gamma} s \bar{t}} h_{\bar{s} t}-\frac{1}{2} R_{\alpha \bar{t}} h_{t \bar{\gamma}}-\frac{1}{2} R_{t \bar{\gamma}} h_{\alpha \bar{t}}\right) \\
& \quad+\nabla_{\gamma} R_{\alpha \bar{p}} h_{p \bar{\gamma}} \\
= & R_{s \bar{t}} \nabla_{t} h_{\alpha \bar{s}}+\nabla_{\alpha} R_{s \bar{t}} h_{\bar{s} t}+R_{\alpha \bar{\gamma} s \bar{t}} \nabla_{\gamma} h_{\bar{s} t}+\frac{1}{2} \nabla_{\gamma} R_{\alpha \bar{t}} h_{t \bar{\gamma}} \\
& \quad-\frac{1}{2} R_{\alpha \bar{t}} \nabla_{\gamma} h_{t \bar{\gamma}}-\frac{1}{2} \nabla_{t} R h_{\alpha \bar{t}}-\frac{1}{2} R_{t \bar{\gamma}} \nabla_{\gamma} h_{\alpha \bar{t}}+\nabla_{\gamma}\left(\Delta h_{\alpha \bar{\gamma}}\right) .
\end{aligned}
$$

Now we calculate $\nabla_{\gamma}\left(\Delta h_{\alpha \bar{\gamma}}\right)$. By definition,

$$
\nabla_{\gamma}\left(\Delta h_{\alpha \bar{\gamma}}\right)=\frac{1}{2} \nabla_{\gamma}\left(\nabla_{s} \nabla_{\bar{s}}+\nabla_{\bar{s}} \nabla_{s}\right) h_{\alpha \bar{\gamma}} .
$$

On the other hand,

$$
\begin{aligned}
\nabla_{\gamma} \nabla_{s} \nabla_{\bar{s}} h_{\alpha \bar{\gamma}} & =\nabla_{s} \nabla_{\gamma} \nabla_{\bar{s}} h_{\alpha \bar{\gamma}} \\
& =\nabla_{s}\left[\nabla_{\bar{s}} \nabla_{\gamma} h_{\alpha \bar{\gamma}}-R_{\alpha \bar{p} \gamma \bar{s}} h_{p \bar{\gamma}}+R_{p \bar{\gamma} \gamma \bar{s}} h_{\alpha \bar{p}}\right] \\
& =\nabla_{s} \nabla_{\bar{s}} \nabla_{\gamma} h_{\alpha \bar{\gamma}}-\nabla_{\gamma} R_{\alpha \bar{p}} h_{p \bar{\gamma}}-R_{\alpha \bar{p} \gamma \bar{s}} \nabla_{s} h_{p \bar{\gamma}}+\nabla_{p} R h_{\alpha \bar{p}}+R_{p \bar{s}} \nabla_{s} h_{\alpha \bar{p}}
\end{aligned}
$$


Similarly,

$$
\begin{aligned}
\nabla_{\gamma} \nabla_{\bar{s}} \nabla_{s} h_{\alpha \bar{\gamma}}= & \nabla_{\bar{s}} \nabla_{\gamma} \nabla_{s} h_{\alpha \bar{\gamma}}+R_{p \bar{\gamma} \gamma \bar{s}} \nabla_{s} h_{\alpha \bar{p}}-R_{s \bar{p} \gamma \bar{s}} \nabla_{p} h_{\alpha \bar{\gamma}} \\
& -R_{\alpha \bar{p} \gamma \bar{s}} \nabla_{s} h_{p \bar{\gamma}} \\
= & \nabla_{\bar{s}} \nabla_{s} \nabla_{\gamma} h_{\alpha \bar{\gamma}}+R_{p \bar{s}} \nabla_{s} h_{\alpha \bar{p}}-R_{\gamma \bar{p}} \nabla_{p} h_{\alpha \bar{\gamma}}-R_{\alpha \bar{p} \gamma \bar{s}} \nabla_{s} h_{p \bar{\gamma}} .
\end{aligned}
$$

Combining the above three we have

$$
\begin{aligned}
\nabla_{\gamma}\left(\Delta h_{\alpha \bar{\gamma}}\right)= & \Delta\left(\nabla_{\gamma} h_{\alpha \bar{\gamma}}\right)-\frac{1}{2} \nabla_{\gamma} R_{\alpha \bar{p}} h_{p \bar{\gamma}}-R_{\alpha \bar{p} \gamma \bar{s}} \nabla_{s} h_{p \bar{\gamma}} \\
& +\frac{1}{2} \nabla_{p} R h_{\alpha \bar{p}}+R_{p \bar{s}} \nabla_{s} h_{\alpha \bar{p}}-\frac{1}{2} R_{\gamma \bar{p}} \nabla_{p} h_{\alpha \bar{\gamma}}
\end{aligned}
$$

Plugging the above into (1.23), the lemma is proved.

LEMMA 1.5. Under normal coordinates at a point,

$$
\begin{aligned}
\left(\frac{\partial}{\partial t}-\Delta\right)\left(g^{\alpha \bar{\beta}} \nabla_{\bar{\beta}} \operatorname{div}(h)_{\alpha}\right)= & R_{s \bar{\alpha}} \nabla_{\bar{s}} \operatorname{div}(h)_{\alpha}+\nabla_{\bar{\alpha}} R_{s \bar{t}} \nabla_{t} h_{\alpha \bar{s}}+\nabla_{\alpha} R_{s \bar{t}} \nabla_{\bar{\alpha}} h_{\bar{s} t} \\
& +R_{s \bar{t}} \nabla_{\bar{\alpha}} \nabla_{t} h_{\alpha \bar{s}}+\left(\nabla_{\bar{\alpha}} \nabla_{\alpha} R_{\bar{s} \bar{t}}\right) h_{\bar{s} t} .
\end{aligned}
$$

Proof. Direct calculation shows that

$$
\begin{aligned}
\frac{\partial}{\partial t}\left(g^{\alpha \bar{\beta}} \nabla_{\bar{\beta}} \operatorname{div}(h)_{\alpha}\right)= & g^{\alpha \bar{t}} R_{s \bar{t}} g^{s \bar{\beta}} \nabla_{\bar{\beta}} \operatorname{div}(h)_{\alpha}+\nabla_{\bar{\alpha}}\left(\frac{\partial}{\partial t} \operatorname{div}(h)_{\alpha}\right) \\
= & R_{s \bar{\alpha}} \nabla_{\bar{s}}\left(\operatorname{div}(h)_{\alpha}\right) \\
& +\nabla_{\bar{\alpha}}\left[\Delta \operatorname{div}(h)_{\alpha}+R_{s \bar{t}} \nabla_{t} h_{\alpha \bar{s}}+\nabla_{\alpha} R_{s \bar{t}} h_{\bar{s} t}-\frac{1}{2} R_{\alpha \bar{t}} \operatorname{div}(h)_{t}\right],
\end{aligned}
$$

by Lemma 1.4. Therefore we have that

$$
\begin{aligned}
\frac{\partial}{\partial t}\left(g^{\alpha \bar{\beta}} \nabla_{\bar{\beta}} \operatorname{div}(h)_{\alpha}\right)= & \nabla_{\bar{\alpha}}\left(\Delta \operatorname{div}(h)_{\alpha}\right)+\frac{1}{2} R_{s \bar{\alpha}} \nabla_{\bar{s}}\left(\operatorname{div}(h)_{\alpha}\right) \\
& +\nabla_{\bar{\alpha}} R_{s \bar{t}} \nabla_{t} h_{\alpha \bar{s}}+\nabla_{\alpha} R_{s \bar{t}} \nabla_{\bar{\alpha}} h_{\bar{s} t} \\
& +R_{s \bar{t}} \nabla_{\bar{\alpha}} \nabla_{t} h_{\alpha \bar{s}}+\nabla_{\bar{\alpha}} \nabla_{\alpha} R_{s \bar{t}} h_{\bar{s} t}-\frac{1}{2} \nabla_{\bar{t}} R\left(\operatorname{div}(h)_{t}\right)
\end{aligned}
$$

Now we calculate $\nabla_{\bar{\alpha}}\left(\Delta \operatorname{div}(h)_{\alpha}\right)$. By definition

$$
\nabla_{\bar{\alpha}}\left(\Delta \operatorname{div}(h)_{\alpha}\right)=\frac{1}{2} \nabla_{\bar{\alpha}} \nabla_{s} \nabla_{\bar{s}} \operatorname{div}(h)_{\alpha}+\frac{1}{2} \nabla_{\bar{\alpha}} \nabla_{\bar{s}} \nabla_{s} \operatorname{div}(h)_{\alpha} .
$$


On the other hand

$$
\begin{aligned}
\nabla_{\bar{\alpha}} \nabla_{\bar{s}} \nabla_{s} \operatorname{div}(h)_{\alpha} & =\nabla_{\bar{s}} \nabla_{\bar{\alpha}} \nabla_{s} \operatorname{div}(h)_{\alpha} \\
& =\nabla_{\bar{s}}\left[\nabla_{s} \nabla_{\bar{\alpha}} \operatorname{div}(h)_{\alpha}+R_{\alpha \bar{p} s \bar{\alpha}} \operatorname{div}(h)_{p}\right] \\
& =\nabla_{\bar{s}} \nabla_{s} \nabla_{\bar{\alpha}} \operatorname{div}(h)_{\alpha}+\left(\nabla_{\bar{s}} R\right)\left(\operatorname{div}(h)_{s}\right)+R_{s \bar{p}} \nabla_{\bar{s}} \operatorname{div}(h)_{p}
\end{aligned}
$$

and

$$
\begin{aligned}
\nabla_{\bar{\alpha}} \nabla_{s} \nabla_{\bar{s}} \operatorname{div}(h)_{\alpha} & =\nabla_{s} \nabla_{\bar{\alpha}} \nabla_{\bar{s}} \operatorname{div}(h)_{\alpha}+R_{\bar{p} s} \nabla_{\bar{s}} \operatorname{div}(h)_{p}-R_{p \bar{\alpha}} \nabla_{\bar{p}} \operatorname{div}(h)_{\alpha} \\
& =\nabla_{s} \nabla_{\bar{s}} \nabla_{\bar{\alpha}} \operatorname{div}(h)_{\alpha}+R_{\bar{p} s} \nabla_{\bar{s}} \operatorname{div}(h)_{p}-R_{p \bar{\alpha}} \nabla_{\bar{p}} \operatorname{div}(h)_{\alpha} .
\end{aligned}
$$

Combining the above three we have that

$$
\nabla_{\bar{\alpha}}\left(\Delta \operatorname{div}(h)_{\alpha}\right)=\Delta\left(\nabla_{\bar{\alpha}} \operatorname{div}(h)_{\alpha}\right)+\frac{1}{2} \nabla_{\bar{s}} R\left(\operatorname{div}(h)_{s}\right)+\frac{1}{2} R_{s \bar{p}} \nabla_{\bar{s}} \operatorname{div}(h)_{p} .
$$

Plugging into (1.25), this completes the proof of Lemma 1.3.

Taking the conjugation we will have the following lemmas.

LEMMA $1.4^{\prime}$. Under normal coordinates at a point,

$$
\left(\frac{\partial}{\partial t}-\Delta\right)\left(\operatorname{div}(h)_{\bar{\beta}}\right)=R_{s \bar{p}} \nabla_{\bar{s}} h_{p \bar{\beta}}+\nabla_{\bar{\beta}} R_{p \bar{\gamma}} h_{\gamma \bar{p}}-\frac{1}{2} R_{t \bar{\beta}} \operatorname{div}(h)_{\bar{t}}
$$

LEMMA 1.5'. Under normal coordinates at a point,

$$
\begin{aligned}
\left(\frac{\partial}{\partial t}-\Delta\right)\left(g^{\beta \bar{\alpha}} \nabla_{\beta} \operatorname{div}(h)_{\bar{\alpha}}\right)= & R_{\alpha \bar{p}} \nabla_{p} \operatorname{div}(h)_{\bar{\alpha}}+\nabla_{\alpha} R_{s \bar{p}} \nabla_{\bar{s}} h_{p \bar{\alpha}} \\
& +\nabla_{\bar{\alpha}} R_{p \bar{\gamma}} \nabla_{\alpha} h_{\gamma \bar{p}} \\
& +R_{s \bar{p}} \nabla_{\alpha} \nabla_{\bar{s}} h_{p \bar{\alpha}}+\left(\nabla_{\alpha} \nabla_{\bar{\alpha}} R_{p \bar{\gamma}}\right) h_{\gamma \bar{p}} .
\end{aligned}
$$

Now we are ready to calculate $\left(\frac{\partial}{\partial t}-\Delta\right) Z$. By Proposition $1.1, h$ is nonnegative. However $h$ may be zero somewhere, we consider $\widehat{Z}$ instead, where:

$$
\widehat{Z}=\mathrm{I}+\mathrm{II}+\mathrm{III}+\mathrm{IV}+\mathrm{V},
$$

where

$$
\begin{aligned}
\mathrm{I} & \left.=\frac{1}{2}\left[g^{\alpha \bar{\beta}} \nabla_{\bar{\beta}} \operatorname{div}(h)_{\alpha}\right)+g^{\gamma \bar{\delta}} \nabla_{\gamma} \operatorname{div}(h)_{\bar{\delta}}\right], \\
\mathrm{II} & =g^{\alpha \bar{\beta}} g^{\gamma \bar{\delta}}\left[R_{\alpha \bar{\delta}} h_{\gamma \bar{\beta}}+\epsilon \mathcal{R}\right],
\end{aligned}
$$




$$
\begin{aligned}
\mathrm{III} & =g^{\alpha \bar{\beta}} \operatorname{div}(h)_{\alpha} V_{\bar{\beta}}+g^{\gamma \bar{\delta}} \operatorname{div}(h)_{\bar{\delta}} V_{\gamma}, \\
\mathrm{IV} & =g^{\alpha \bar{\beta}} g^{\gamma \bar{\delta}} \widetilde{h}_{\alpha \bar{\delta}} V_{\bar{\beta}} V_{\alpha}, \\
\mathrm{V} & =\frac{H+\epsilon m}{t}
\end{aligned}
$$

and where $\epsilon>0$ is a fixed constant, $\mathcal{R}$ is the scalar curvature, and $\widetilde{h}_{\alpha \bar{\beta}}=$ $h_{\alpha \bar{\beta}}+\epsilon g_{\alpha \bar{\beta}}$. We calculate them one by one. In the following, we always do computations in normal coordinates at a point because the final result will not depend on the choice of coordinates.

From Lemma 1.5, Lemma $1.5^{\prime}$, and the second Bianchi identity we have that

$$
\begin{aligned}
\left(\frac{\partial}{\partial t}-\Delta\right) \mathrm{I}= & \frac{1}{2}\left[R_{\alpha \bar{p}} \nabla_{p} \operatorname{div}(h)_{\bar{\alpha}}+R_{p \bar{\alpha}} \nabla_{\bar{p}} \operatorname{div}(h)_{\alpha}\right] \\
+ & \frac{1}{2}\left[R_{s \bar{p}} \nabla_{\alpha} \nabla_{\bar{s}} h_{p \bar{\alpha}}+R_{s \bar{p}} \nabla_{\bar{\alpha}} \nabla_{p} h_{\alpha \bar{s}}\right] \\
+ & \Delta R_{s \bar{t}} h_{\bar{s} t}+\nabla_{\bar{t}} R_{s \bar{\alpha}} \nabla_{t} h_{\alpha \bar{s}}+\nabla_{t} R_{s \bar{\alpha}} \nabla_{\bar{t}} h_{\bar{s} \alpha .} \\
\left(\frac{\partial}{\partial t}-\Delta\right) \mathrm{II}= & \left(\frac{\partial}{\partial t}-\Delta\right)\left(g^{\alpha \bar{\delta}} g{ }^{\gamma \bar{\beta}} R_{\alpha \bar{\beta}} h_{\gamma \bar{\delta}}+\epsilon \mathcal{R}\right) \\
= & 2 R_{\beta \bar{\gamma}} R_{\alpha \bar{\beta}} h_{\gamma \bar{\alpha}}+\left(\left(\frac{\partial}{\partial t}-\Delta\right) R_{\alpha \bar{\beta}}\right) h_{\beta \bar{\alpha}} \\
& +R_{\alpha \bar{\beta}}\left(\left(\frac{\partial}{\partial t}-\Delta\right) h_{\beta \bar{\alpha}}\right) \\
& -\nabla_{s} R_{\alpha \bar{\beta}} \nabla_{\bar{s}} h_{\beta \bar{\alpha}}-\nabla_{\bar{s}} R_{\alpha \bar{\beta}} \nabla_{s} h_{\beta \bar{\alpha}}+\epsilon\left|R_{\alpha \bar{\beta}}\right|^{2} \\
= & 2 R_{\alpha \bar{\beta} s \bar{t}} R_{\bar{s} t} h_{\beta \bar{\alpha}}-\nabla_{s} R_{\alpha \bar{\beta}} \nabla_{\bar{s}} h_{\beta \bar{\alpha}} \\
& -\nabla_{\bar{s}} R_{\alpha \bar{\beta}} \nabla_{s} h_{\beta \bar{\alpha}}+\epsilon\left|R_{\alpha \bar{\beta}}\right|^{2} .
\end{aligned}
$$

Here we have used (1.2) and the equation satisfies by the Ricci form [Sh3]:

$$
\left(\frac{\partial}{\partial t}-\Delta\right) R_{\alpha \bar{\beta}}=R_{\alpha \bar{\beta} \gamma \bar{\delta}} R_{\bar{\gamma} \delta}-R_{\alpha \bar{s}} R_{s \bar{\beta}}
$$

Using Lemma 1.4, Lemma $1.4^{\prime}$ and the second Bianchi identity we have that

$$
\begin{aligned}
\left(\frac{\partial}{\partial t}-\Delta\right) \mathrm{III}= & \left(\frac{\partial}{\partial t}-\Delta\right)\left[g^{\alpha \bar{\beta}}\left(\operatorname{div}(h)_{\alpha} V_{\bar{\beta}}+\operatorname{div}(h)_{\bar{\beta}} V_{\alpha}\right)\right] \\
= & R_{\bar{\alpha} \beta} \operatorname{div}(h)_{\alpha} V_{\bar{\beta}}+R_{\bar{\alpha} \beta} \operatorname{div}(h)_{\bar{\beta}} V_{\alpha}+\operatorname{div}(h)_{\alpha} \\
& \times\left(\left(\frac{\partial}{\partial t}-\Delta\right) V_{\bar{\alpha}}\right)+\operatorname{div}(h)_{\bar{\alpha}}\left(\left(\frac{\partial}{\partial t}-\Delta\right) V_{\alpha}\right)
\end{aligned}
$$




$$
\begin{aligned}
& +\left(R_{s \bar{t}} \nabla_{t} h_{\alpha \bar{s}}+\nabla_{s} R_{\alpha \bar{t}} h_{\bar{s} t}-\frac{1}{2} R_{\alpha \bar{t}} \operatorname{div}(h)_{t}\right) V_{\bar{\alpha}} \\
& +\left(R_{s \bar{t}} \nabla_{\bar{s}} h_{t \bar{\alpha}}+\nabla_{\bar{s}} R_{t \bar{\alpha}} h_{s \bar{t}}-\frac{1}{2} R_{t \bar{\alpha}} \operatorname{div}(h)_{\bar{t}}\right) V_{\alpha} \\
& -\nabla_{s} \operatorname{div}(h)_{\alpha} \nabla_{\bar{s}} V_{\bar{\alpha}}-\nabla_{\bar{s}} \operatorname{div}(h)_{\alpha} \nabla_{s} V_{\bar{\alpha}} \\
& -\nabla_{s} \operatorname{div}(h)_{\bar{\alpha}} \nabla_{\bar{s}} V_{\alpha}-\nabla_{\bar{s}} \operatorname{div}(h)_{\bar{\alpha}} \nabla_{s} V_{\alpha} .
\end{aligned}
$$

Using (1.2) we have

$$
\begin{aligned}
\left(\frac{\partial}{\partial t}-\Delta\right) \mathrm{IV}= & \left(\frac{\partial}{\partial t}-\Delta\right)\left(g^{\alpha \bar{\beta}} g \bar{\delta} \widetilde{h}_{\alpha \bar{\delta}} V_{\gamma} V_{\bar{\beta}}\right) \\
= & R_{\alpha \bar{\beta} s \bar{t}} h_{\bar{s} t} V_{\beta} V_{\bar{\alpha}}+\frac{1}{2} R_{\alpha \bar{s}} h_{s \bar{\gamma}} V_{\gamma} V_{\bar{\alpha}}+\frac{1}{2} h_{\alpha \bar{s}} R_{s \bar{\gamma}} V_{\gamma} V_{\bar{\alpha}} \\
& +\widetilde{h}_{\alpha \bar{\gamma}}\left(\left(\frac{\partial}{\partial t}-\Delta\right) V_{\gamma}\right) V_{\bar{\alpha}}+\widetilde{h}_{\alpha \bar{\gamma}} V_{\gamma}\left(\left(\frac{\partial}{\partial t}-\Delta\right) V_{\bar{\alpha}}\right) \\
& -\nabla_{s} h_{\alpha \bar{\gamma}} \nabla_{\bar{s}}\left(V_{\gamma} V_{\bar{\alpha}}\right)-\nabla_{\bar{s}} h_{\alpha \bar{\gamma}} \nabla_{s}\left(V_{\gamma} V_{\bar{\alpha}}\right) \\
& -\widetilde{h}_{\alpha \bar{\gamma}}\left[\nabla_{s} V_{\gamma} \nabla_{\bar{s}} V_{\bar{\alpha}}+\nabla_{\bar{s}} V_{\gamma} \nabla_{s} V_{\bar{\alpha}}+\epsilon R_{\alpha \bar{\gamma}} V_{\bar{\alpha}} V_{\gamma}\right] .
\end{aligned}
$$

Taking trace on (1.2) one can have

$$
\left(\frac{\partial}{\partial t}-\Delta\right) \mathrm{V}=\frac{R_{\alpha \bar{s}} h_{s \bar{\alpha}}}{t}-\frac{H+\epsilon m}{t^{2}}
$$

Now combining them together we have that

$$
\begin{aligned}
\left(\frac{\partial}{\partial t}-\Delta\right) Z= & Y_{1}+\frac{1}{2}\left[R_{\alpha \bar{p}} \nabla_{p} \operatorname{div}(h)_{\bar{\alpha}}+R_{p \bar{\alpha}} \nabla_{\bar{p}} \operatorname{div}(h)_{\alpha}\right] \\
& +\frac{1}{2}\left[R_{s \bar{p}} \nabla_{\alpha} \nabla_{\bar{s}} h_{p \bar{\alpha}}+R_{s \bar{p}} \nabla_{\bar{\alpha}} \nabla_{p} h_{\alpha \bar{s}}\right] \\
& +R_{\alpha \bar{\beta} s \bar{t}} R_{\bar{\alpha} \beta} h_{t \bar{s}}+R_{\bar{\alpha} \beta} \operatorname{div}(h)_{\alpha} V_{\bar{\beta}}+R_{\bar{\alpha} \beta} \operatorname{div}(h)_{\bar{\beta}} V_{\alpha} \\
& +\operatorname{div}(h)_{\alpha}\left(\left(\frac{\partial}{\partial t}-\Delta\right) V_{\bar{\alpha}}\right)+\operatorname{div}(h)_{\bar{\alpha}}\left(\left(\frac{\partial}{\partial t}-\Delta\right) V_{\alpha}\right) \\
& -\nabla_{s} \operatorname{div}(h)_{\alpha} \nabla_{\bar{s}} V_{\bar{\alpha}}-\nabla_{\bar{s}} \operatorname{div}(h)_{\alpha} \nabla_{s} V_{\bar{\alpha}} \\
& -\nabla_{s} \operatorname{div}(h)_{\bar{\alpha}} \nabla_{\bar{s}} V_{\alpha}-\nabla_{\bar{s}} \operatorname{div}(h)_{\bar{\alpha}} \nabla_{s} V_{\alpha} \\
& +R_{s \bar{t}} \nabla_{t} h_{\alpha \bar{s}} V_{\bar{\alpha}}-\frac{1}{2} R_{\alpha \bar{t}} \operatorname{div}(h)_{t} V_{\bar{\alpha}}+R_{s \bar{t}} \nabla_{\bar{s}} h_{t \bar{\alpha}} V_{\alpha} \\
& -\frac{1}{2} R_{t \bar{\alpha}} \operatorname{div}(h)_{\bar{t}} V_{\alpha} \\
& +\frac{1}{2} R_{\alpha \bar{s}} h_{s \bar{\gamma}} V_{\gamma} V_{\bar{\alpha}}+\frac{1}{2} h_{\alpha \bar{s}} R_{s \bar{\gamma}} V_{\gamma} V_{\bar{\alpha}}
\end{aligned}
$$




$$
\begin{aligned}
& +\widetilde{h}_{\alpha \bar{\gamma}}\left(\left(\frac{\partial}{\partial t}-\Delta\right) V_{\gamma}\right) V_{\bar{\alpha}}+\widetilde{h}_{\alpha \bar{\gamma}} V_{\gamma}\left(\left(\frac{\partial}{\partial t}-\Delta\right) V_{\bar{\alpha}}\right) \\
& -\nabla_{s} h_{\alpha \bar{\gamma}} \nabla_{\bar{s}}\left(V_{\gamma} V_{\bar{\alpha}}\right)-\nabla_{\bar{s}} h_{\alpha \bar{\gamma}} \nabla_{s}\left(V_{\gamma} V_{\bar{\alpha}}\right) \\
& -\widetilde{h}_{\alpha \bar{\gamma}}\left[\nabla_{s} V_{\gamma} \nabla_{\bar{s}} V_{\bar{\alpha}}+\nabla_{\bar{s}} V_{\gamma} \nabla_{s} V_{\bar{\alpha}}\right]-\frac{H+\epsilon m}{t^{2}} \\
& +\epsilon\left|R_{\alpha \bar{\beta}}\right|^{2}+\epsilon R_{\alpha \bar{\gamma}} V_{\bar{\alpha}} V_{\gamma},
\end{aligned}
$$

where

$$
Y_{1}=\left[\Delta R_{s \bar{t}}+R_{s \bar{\alpha} \alpha \bar{\beta}} R_{\bar{\alpha} \beta}+\nabla_{\alpha} R_{s \bar{t}} V_{\bar{\alpha}}+\nabla_{\bar{\alpha}} R_{s \bar{t}} V_{\alpha}+R_{s \bar{t} \alpha \bar{\beta}} V_{\bar{\alpha}} V_{\beta}+\frac{R_{s \bar{t}}}{t}\right] h_{\bar{s} t} .
$$

By Proposition 1.1, $h_{\alpha \bar{\beta}} \geq 0$ on $M \times[0, T]$. Hence by Cao's LYH inequality [Co1-2] and the fact that $\left(M, g_{\alpha \bar{\beta}}(x, t)\right)$ has nonnegative holomorphic bisectional curvature, the two factors in $Y_{1}$ are all nonnegative tensors. Therefore $Y_{1} \geq 0$. Since $\widetilde{h}_{\alpha \bar{\beta}} \geq \epsilon g_{\alpha \bar{\beta}}$, for each $(x, t), \widehat{Z}$ attains minimum for some $V$. Then by the first variation we have

$$
\operatorname{div}(h)_{\alpha}+\widetilde{h}_{\alpha \bar{\gamma}} V_{\gamma}=0 \quad \text { and } \quad \operatorname{div}(h)_{\bar{\alpha}}+\widetilde{h}_{\gamma \bar{\alpha}} V_{\bar{\gamma}}=0
$$

Direct calculation also shows that

$$
\begin{aligned}
R_{p \bar{\alpha}} \nabla_{\bar{p}} \operatorname{div}(h)_{\alpha}+R_{\alpha \bar{p}} \nabla_{p} \operatorname{div}(h)_{\bar{\alpha}}= & R_{s \bar{p}} \nabla_{\alpha} \nabla_{\bar{s}} h_{p \bar{\alpha}}+R_{s \bar{p}} \nabla_{\bar{\alpha}} \nabla_{p} h_{\alpha \bar{s}} \\
& +2 R_{\alpha \bar{p}} R_{p \bar{\alpha} s \bar{\gamma}} h_{\gamma \bar{s}}-2 R_{\alpha \bar{p}} R_{p \bar{s}} h_{s \bar{\alpha}} .
\end{aligned}
$$

Combining (1.34)-(1.37) we have that

$$
\begin{aligned}
\left(\frac{\partial}{\partial t}-\Delta\right) \widehat{Z}= & Y_{1}+\left[R_{\alpha \bar{p}} \nabla_{p} \operatorname{div}(h)_{\bar{\alpha}}+R_{p \bar{\alpha}} \nabla_{\bar{p}} \operatorname{div}(h)_{\alpha}\right]+R_{\alpha \bar{p}} R_{p \bar{s}} h_{s \bar{\alpha}} \\
& -\nabla_{s} \operatorname{div}(h)_{\alpha} \nabla_{\bar{s}} V_{\bar{\alpha}}-\nabla_{\bar{s}} \operatorname{div}(h)_{\alpha} \nabla_{s} V_{\bar{\alpha}} \\
& -\nabla_{s} \operatorname{div}(h)_{\bar{\alpha}} \nabla_{\bar{s}} V_{\alpha}-\nabla_{\bar{s}} \operatorname{div}(h)_{\bar{\alpha}} \nabla_{s} V_{\alpha} \\
& +R_{s \bar{t}} \nabla_{t} h_{\alpha \bar{s}} V_{\bar{\alpha}}+R_{s \bar{t}} \nabla_{\bar{s}} h_{t \bar{\alpha}} V_{\alpha} \\
& -\nabla_{s} h_{\alpha \bar{\gamma}} \nabla_{\bar{s}}\left(V_{\gamma} V_{\bar{\alpha}}\right)-\nabla_{\bar{s}} h_{\alpha \bar{\gamma}} \nabla_{s}\left(V_{\gamma} V_{\bar{\alpha}}\right) \\
& -\widetilde{h}_{\alpha \bar{\gamma}}\left[\nabla_{s} V_{\gamma} \nabla_{\bar{s}} V_{\bar{\alpha}}+\nabla_{\bar{s}} V_{\gamma} \nabla_{s} V_{\bar{\alpha}}\right]-\frac{H+\epsilon m}{t^{2}} .
\end{aligned}
$$

Differentiate (1.36) and we have

$$
\begin{aligned}
& \nabla_{s} \operatorname{div}(h)_{\alpha}+\left(\nabla_{s} h_{\alpha \bar{\gamma}}\right) V_{\gamma}+\widetilde{h}_{\alpha \bar{\gamma}} \nabla_{s} V_{\gamma}=0, \\
& \nabla_{s} \operatorname{div}(h)_{\bar{\alpha}}+\left(\nabla_{s} h_{\gamma \bar{\alpha}}\right) V_{\bar{\gamma}}+\widetilde{h}_{\gamma \bar{\alpha}} \nabla_{s} V_{\bar{\gamma}}=0,
\end{aligned}
$$




$$
\begin{aligned}
& \nabla_{\bar{s}} \operatorname{div}(h)_{\alpha}+\left(\nabla_{\bar{s}} h_{\alpha \bar{\gamma}}\right) V_{\gamma}+\widetilde{h}_{\alpha \bar{\gamma}} \nabla_{\bar{s}} V_{\gamma}=0, \\
& \nabla_{\bar{s}} \operatorname{div}(h)_{\bar{\alpha}}+\left(\nabla_{\bar{s}} h_{\gamma \bar{\alpha}}\right) V_{\bar{\gamma}}+\widetilde{h}_{\gamma \bar{\alpha}} \nabla_{\bar{s}} V_{\bar{\gamma}}=0 .
\end{aligned}
$$

Plugging the above into (1.34) we have that

$$
\begin{aligned}
\left(\frac{\partial}{\partial t}-\Delta\right) \widehat{Z}= & Y_{1}+R_{\alpha \bar{p}} R_{p \bar{s}} h_{s \bar{\alpha}}-R_{\alpha \bar{p}} h_{\gamma \bar{\alpha}} \nabla_{p} V_{\bar{\gamma}}-R_{p \bar{\alpha}} h_{\alpha \bar{\gamma}} \nabla_{\bar{p}} V_{\gamma} \\
& +\widetilde{h}_{\gamma \bar{\alpha}} \nabla_{s} V_{\bar{\gamma}} \nabla_{\bar{s}} V_{\alpha}+\widetilde{h}_{\gamma \bar{\alpha}} \nabla_{\bar{s}} V_{\bar{\gamma}} \nabla_{s} V_{\alpha} \\
& -\frac{H+\epsilon m}{t^{2}}+\epsilon\left|R_{\alpha \bar{\beta}}\right|^{2}
\end{aligned}
$$

Let

$$
\begin{aligned}
Y_{2}= & \widetilde{h}_{\gamma \bar{\alpha}}\left[\nabla_{p} V_{\bar{\gamma}}-R_{p \bar{\gamma}}-\frac{1}{t} g_{p \bar{\gamma}}\right]\left[\nabla_{\bar{p}} V_{\alpha}-R_{\alpha \bar{p}}-\frac{1}{t} g_{\bar{p} \alpha}\right] \\
& +\widetilde{h}_{\gamma \bar{\alpha}} \nabla_{\bar{p}} V_{\bar{\gamma}} \nabla_{p} V_{\alpha} .
\end{aligned}
$$

By Proposition 1.1 again, $Y_{2} \geq 0$.

$$
\begin{aligned}
\left(\frac{\partial}{\partial t}-\Delta\right) \widehat{Z}=Y_{1}+Y_{2}-\frac{1}{t}[ & -\widetilde{h}_{\gamma \bar{\alpha}} \nabla_{\alpha} V_{\bar{\gamma}}-\widetilde{h}_{\gamma \bar{\alpha}} \nabla_{\bar{\alpha}} V_{\gamma} \\
& \left.+2 R_{\alpha \bar{\gamma}} h_{\gamma \bar{\alpha}}+\frac{2(H+\epsilon m)}{t}+2 \epsilon \mathcal{R}\right] .
\end{aligned}
$$

Using (1.36) we also know that

$$
\widehat{Z}=R_{\alpha \bar{\beta}} h_{\bar{\alpha} \beta}-\frac{1}{2} \widetilde{h}_{\alpha \bar{\beta}} \nabla_{\bar{\alpha}} V_{\beta}-\frac{1}{2} \widetilde{h}_{\beta \bar{\alpha}} \nabla_{\alpha} V_{\bar{\beta}}+\frac{H+\epsilon m}{t}+\epsilon \mathcal{R}
$$

Plugging into (1.42) and using the fact that $Y_{1} \geq 0$ and $Y_{2} \geq 0$, we have

$$
\left(\frac{\partial}{\partial t}-\Delta\right) \widehat{Z} \geq-\frac{2 \widehat{Z}}{t}
$$

where $V$ is the smooth vector field given by (1.36). Note that both sides of (1.44) do not depend on the choice of coordinates.

Proof of Theorem 1.2. Since $\widetilde{h}_{\alpha \bar{\beta}} \geq \epsilon g_{\alpha \bar{\beta}}$ on $M \times[0, T]$, by (1.36) and (1.39), we have

$$
\|V\| \leq C_{1}\|\nabla h\|
$$


and

$$
\|\nabla V\| \leq C_{2}\left(\|\nabla \nabla h\|+\|\nabla h\|^{2}\right)
$$

for some constants $C_{1}$ and $C_{2}$. Combining this with (1.44), we have

$$
\left|t^{2} \widehat{Z}\right|^{2} \leq C_{3}\left(\Phi+\Phi\left(\Psi^{2}+\Lambda\right)+1\right)
$$

for some constant $C_{3}$. By (1.43), the corresponding $\widehat{Z}$ satisfies

$$
\left(\frac{\partial}{\partial t}-\Delta\right)\left(t^{2} \widehat{Z}\right) \geq 0
$$

for the vector field which minimizes $\widehat{Z}$. By Lemma 1.2, Lemma 1.3, and (1.45), we have

$$
\int_{0}^{T} \int_{M} \exp \left(-a r_{0}^{2}(x)\right)\left(t^{2} \widehat{Z}\right)^{2} d V_{t} d t<\infty
$$

for any $a>0$. By the maximum principle Theorem 1.1, we have $t^{2} \widehat{Z} \geq 0$ because it is obvious that $t^{2} \widetilde{Z}=0$ at $t=0$. Since this is true for the vector field $V$ minimizing $\widehat{Z}$, we have $\widehat{Z} \geq 0$ for any $(1,0)$ vector field. Let $\epsilon \rightarrow 0$ and the proof of the theorem is completed.

Remark 1.1. (i) The theorem is still true for the case that $M$ is compact with positive holomorphic bisectional curvature because of the result in [Co1]. (ii) When $h_{\alpha \bar{\beta}}=R_{\alpha \bar{\beta}}$, it is known that the Ricci tensor satisfies (1.2). Therefore we can apply Theorem 1.1 to this case. Since

$$
\operatorname{div}(h)_{\alpha}=\nabla_{\gamma} R_{\alpha \bar{\gamma}}=\nabla_{\alpha} \mathcal{R} \quad \text { and } \quad \operatorname{div}(h)_{\bar{\delta}}=\nabla_{\bar{\alpha}} R_{\alpha \bar{\delta}}=\nabla_{\bar{\delta}} \mathcal{R}
$$

we have

$$
Z=\Delta \mathcal{R}+R_{\alpha \bar{\beta}} R_{\bar{\alpha} \beta}+\nabla_{\alpha} \mathcal{R} V_{\bar{\alpha}}+\nabla_{\bar{\alpha}} \mathcal{R} V_{\alpha}+R_{\alpha \bar{\beta}} V_{\bar{\alpha}} V_{\beta}+\frac{\mathcal{R}}{t} \geq 0
$$

It is the trace of the LYH inequality proved by Cao in [Co1-2]. Hence Theorem 1.1 can be considered as a generalization of the LYH inequality of Cao for the scalar curvature. However, we should emphasize that Cao's result has been used in the proof of Theorem 1.2.

2. Deforming plurisubharmonic functions. Let $\left(M^{m}, g_{\alpha \bar{\beta}}(x, t)\right)$ be a complete noncompact Kähler manifold with bounded nonnegative holomorphic bisectional curvature deformed by the Kähler-Ricci flow (1.1). As in the previous section we assume that (1.1) has solution on $M \times[0, T]$ which satisfies condi- 
tions (i)-(iv) in that section. In this section we shall study the plurisubharmonic functions deformed by the time-dependent heat equation:

$$
\left\{\begin{array}{l}
\left(\frac{\partial}{\partial t}-\Delta\right) u(x, t)=0, \\
u(x, 0)=u_{0}(x)
\end{array}\right.
$$

where $\Delta=g^{\alpha \bar{\beta}}(x, t) \frac{\partial^{2}}{\partial z_{\alpha} \partial \bar{z}_{\beta}}$ and $u_{0}(x)$ is a plurisubharmonic function on $M$.

First, we shall consider the more general case and drop the assumption that $u_{0}$ is plurisubharmonic. We have the following existence result.

Proposition 2.1. Let $u_{0}$ be a continuous function such that $\left|u_{0}(x)\right| \leq$ $\exp \left(a\left(r_{0}(x)+1\right)\right)$ for all $x$ for some positive constant $a>0$. Then there is a unique solution of (2.1) on $M \times[0, T]$ such that

$$
|u(x, t)| \leq \exp \left(b\left(r_{0}(x)+1\right)\right)
$$

on $M \times[0, T]$ for some positive constant $b$.

Proof By Lemma 1.1, there exists a function $\varphi(x)$ such that

$$
\exp \left(b\left(r_{0}(x)+1\right)\right) \geq \varphi(x) \geq \exp \left(a\left(r_{0}(x)+1\right)\right)
$$

for some positive constant and $b$ for all $(x, t) \in M \times[0, T]$, and

$$
\left(\frac{\partial}{\partial t}-\Delta\right) \varphi \geq 0
$$

Using $\varphi$ and $-\varphi$ as barriers, the existence part of the proposition follows. Uniqueness follows from the maximum principle Theorem 1.1.

Next we shall study properties of the solution $u$ obtained in the proposition. We need the following lemma.

Lemma 2.1. Let $u(x, t)$ be a solution of (2.1). Then $u_{\alpha \bar{\beta}}$ satisfies the complex Lichnerowicz heat equation (1.2).

Proof. Differentiate (2.1) and we have

$$
\left(u_{t}\right)_{\gamma \bar{\delta}}=R_{\beta \bar{\alpha} \gamma \bar{\delta}} u_{\alpha \bar{\beta}}+g^{\alpha \bar{\beta}} u_{\alpha \bar{\beta} \gamma \bar{\delta}} .
$$

By definition $\Delta u_{\alpha \bar{\beta}}=\frac{1}{2}\left(u_{\alpha \bar{\beta}, \gamma \bar{\gamma}}+u_{\alpha \bar{\beta}, \bar{\gamma} \gamma}\right)$, in normal coordinates at a point. We need to calculate the difference between the partial derivative $u_{\alpha \bar{\beta} \gamma \bar{\delta}}$ and the covariant derivative $u_{\alpha \bar{\beta}, \gamma \bar{\delta}}$. Direct computations show that, for normal coordinates at a point,

$$
u_{\gamma \bar{\delta}, \alpha \bar{\beta}}=u_{\gamma \bar{\delta} \alpha \bar{\beta}}+u_{s \bar{\delta}} R_{\alpha \bar{\beta} \gamma \bar{s}} .
$$


Using the fact that

$$
u_{\gamma \bar{\delta}, \alpha \bar{\alpha}}=u_{\gamma \bar{\delta}, \bar{\alpha} \alpha}+R_{\gamma \bar{p}} u_{p \bar{\delta}}-R_{p \bar{\delta}} u_{\gamma \bar{p}}
$$

we have

$$
\begin{aligned}
\Delta u_{\gamma \bar{\delta}} & =\frac{1}{2}\left(u_{\gamma \bar{\delta}, \alpha \bar{\alpha}}+u_{\gamma \bar{\gamma}, \bar{\alpha} \alpha}\right) \\
& =u_{\gamma \bar{\delta} \alpha \bar{\alpha}}+\frac{1}{2}\left(R_{\gamma \bar{p}} u_{p \bar{\delta}}+R_{p \bar{\delta}} u_{\gamma \bar{p}}\right)
\end{aligned}
$$

Combining with (2.3), we conclude that $u_{\alpha \bar{\beta}}$ satisfies (1.2).

In the following, $\widetilde{\nabla}$ and $\widetilde{\Delta}$ denote the covariant derivative and the Laplacian with respect to the initial metric.

PROPOSITION 2.2. Let $u_{0}$ be a smooth function such that $\left|u_{0}(x)\right| \leq \exp \left(a\left(r_{0}(x)+\right.\right.$ 1)) for all $x$ for some positive constant $a>0$. Let $u(x, t)$ be the solution of (2.1) obtained in Proposition 2.1. We have the following:

(i) For any $b>0$

$$
\int_{0}^{T} \int_{M} \exp \left(-b r_{0}^{2}(x)\right)\left(|\nabla u|^{2}(x, t)+t\left\|u_{\alpha \bar{\beta}}\right\|^{2}(x, t)\right) d V_{t} d t<\infty,
$$

where $\left\|u_{\alpha \bar{\beta}}\right\|^{2}=g^{\alpha \bar{\delta}} g^{\gamma \bar{\beta}} u_{\alpha \bar{\beta}} u_{\gamma \bar{\delta}}$.

(ii) If in addition, $\int_{B_{0}(o, r)}\left|\widetilde{\nabla} u_{0}\right|^{2} d V_{0} \leq \exp \left(a^{\prime}(1+r)\right)$ for some $a^{\prime}>0$, where $B_{0}(o, r)$ is the geodesic ball with center at o and radius $r$ with respect to the initial metric $g(0)$, then

$$
\int_{0}^{T} \int_{M} \exp \left(-b r_{0}^{2}(x)\right)\left\|u_{\alpha \bar{\beta}}\right\|^{2}(x, t) d V_{t} d t<\infty,
$$

(iii) If in addition $\left|\widetilde{\nabla} u_{0}\right|^{2} \leq C_{1}$ on $M$ then

$$
|\nabla u|^{2} \leq C_{1}
$$

and

$$
\left\|u_{\alpha \bar{\beta}}\right\|^{2}(x, t) \leq \frac{C_{2}}{t}
$$

for some constant $C_{2}$ on $M \times[0, T]$.

Proof. By Proposition 2.1, there exist a positive constant and $c$ such that

$$
|u(x, t)| \leq \exp \left(c\left(r_{0}(x)+1\right)\right)
$$


on $M \times[0, T]$. Since

$$
\left(\frac{\partial}{\partial t}-\Delta\right) u^{2}=-|\nabla u|^{2}
$$

we can proceed as in the proof of Lemma 1.1 to conclude that for any $b>0$

$$
\int_{0}^{T} \int_{M} \exp \left(-b r_{0}^{2}(x)\right)|\nabla u|^{2} d V_{t} d t<\infty .
$$

Direct computations show (see [N-T, Lemma 1.1] for example)

$$
\left(\frac{\partial}{\partial t}-\Delta\right)|\nabla u|^{2}=-\left\|u_{\alpha \beta}\right\|^{2}-\left\|u_{\alpha \bar{\beta}}\right\|^{2} .
$$

Combining with (2.12), one can also proceed as in the proof of Lemma 1.1 and conclude that (2.7) is true. In case $\left|\widetilde{\nabla} u_{0}\right|^{2}$ satisfies the condition in (ii), then one can prove (2.8) similarly.

By (2.13), it is easy to see that $\left(\frac{\partial}{\partial t}-\Delta\right)\left(\sqrt{|\nabla u|^{2}+1}\right) \leq 0$. Suppose $|\widetilde{\nabla} u|^{2} \leq C_{1}$ on $M$, then by (2.7) we can apply Theorem 1.1 to conclude that (2.9) is true.

Since $u_{\alpha \bar{\beta}}$ satisfies (1.2), as in the proof of (1.11) we have

$$
\left(\frac{\partial}{\partial t}-\Delta\right)(1+t \Phi)^{\frac{1}{2}} \leq C_{3} \Phi
$$

on $M \times[0, T]$ for some constant $C_{3}>0$, where $\Phi=\left\|u_{\alpha \bar{\beta}}\right\|^{2}$. Hence on $M \times[0, T]$,

$$
\left(\frac{\partial}{\partial t}-\Delta\right)\left(C_{3}|\nabla u|^{2}+(1+t \Phi)^{\frac{1}{2}}\right) \leq 0
$$

where we have used (2.13). By (2.8) and (2.9), we can apply the maximum principle in $[\mathrm{N}-\mathrm{T}]$ and conclude that

$$
\sup _{M \times[0, T]}\left(C_{3}|\nabla u|^{2}+(1+t \Phi)^{\frac{1}{2}}\right) \leq C_{3} C_{1}+1
$$

where we have used the fact that $|\widetilde{\nabla} u|^{2} \leq C_{1}$. From this (2.10) follows.

Next, we shall study the properties of $u(x, t)$ in case the initial value $u_{0}$ is plurisubharmonic.

THEOREM 2.1. Let $u_{0}(x)$ be a smooth function on $M$ such that $(a) u_{0}$ is plurisubharmonic; and (b) there exists $a>0$ such that $\left|u_{0}(x)\right| \leq \exp \left(a\left(1+r_{0}(x)\right)\right.$ and $\widetilde{\Delta} u_{0} \leq \exp \left(a\left(1+r_{0}(x)\right)\right.$. Let $u$ be the solution of (2.1) obtained in Proposition 2.1. We have the following:

(i) $u(x, t)$ is plurisubharmonic for $t>0$. 
(ii) If $u_{0}$ is not harmonic, then $w=u_{t}>0$ for $t>0$, and we have the following differential inequality:

$$
w_{t}-\frac{|\nabla w|^{2}}{w}+\frac{w}{t} \geq 0
$$

for $t>0$.

If in addition, $\sup _{M}\left|\widetilde{\nabla} u_{0}\right|^{2} \leq C_{1}<\infty$ for some constant $C_{1}$, then $u_{\alpha \bar{\beta}}$ satisfies (2.10) for some constant $C_{2}$.

Proof. Let $f=\widetilde{\Delta} u_{0} \geq 0$. By assumptions, $\left|u_{0}(x)\right| \leq \exp \left(a\left(1+r_{0}(x)\right)\right.$ and $f(x) \leq \exp \left(a\left(1+r_{0}(x)\right)\right.$. It is easy to see that

$$
\int_{B_{0}(o, r)}\left|\widetilde{\nabla} u_{0}\right|^{2} d V_{0} \leq \exp \left(a^{\prime}\left(1+r_{0}(x)\right)\right.
$$

for some $a^{\prime}>0$. Hence $u_{\alpha \bar{\beta}}$ satisfies (2.8) by Proposition 2.2. Since $u_{0}$ is plurisubharmonic, we also have $\left\|u_{\alpha \bar{\beta}}\right\|^{2}(x, 0) \leq \exp \left(a^{\prime \prime}\left(1+r_{0}(x)\right)\right.$ for some $a^{\prime \prime}>0$. By (i), Proposition 1.1 and Lemma 2.1, we conclude that $u$ is plurisubharmonic for $t>0$.

Since $u_{\alpha \bar{\beta}}$ satisfies (1.2) by Lemma 2.1 and $w=u_{t}=\Delta u$, taking trace of (1.2), we have

$$
\left(\frac{\partial}{\partial t}-\Delta\right) w=R_{\alpha \bar{\beta}} u_{\beta \bar{\alpha}} \geq 0
$$

If $w(x, t)=0$ for some $x$ and $t>0$, then by the strong maximum principle (see [Cw3, Proposition 3.6]), we have $\widetilde{\Delta} u_{0}=0$ on $M$. Hence if $u_{0}$ is not harmonic, then $w>0$ for $t>0$.

Since $u_{\alpha \bar{\beta}}$ satisfies the conditions in Theorem 1.2, if we let $h_{\alpha \bar{\beta}}$, in Theorem 1.2 to be $u_{\alpha \bar{\beta}}$, then in normal coordinates

$$
\operatorname{div}(h)_{\alpha}=\nabla_{\gamma} u_{\alpha \bar{\gamma}}=\nabla_{\alpha}\left(u_{t}\right) \quad \text { and } \quad \operatorname{div}(h)_{\bar{\delta}}=\nabla_{\bar{\alpha}} u_{\alpha \bar{\delta}}=\nabla_{\bar{\delta}}\left(u_{t}\right)
$$

and

$$
Z=\Delta\left(u_{t}\right)+R_{\alpha \bar{\beta}} u_{\bar{\alpha} \beta}+\nabla_{\bar{\alpha}}\left(u_{t}\right) V_{\alpha}+\nabla_{\alpha}\left(u_{t}\right) V_{\bar{\alpha}}+u_{\alpha \bar{\beta}} V_{\bar{\alpha}} V_{\beta}+\frac{u_{t}}{t} \geq 0
$$

for any $(1,0)$ vector field $V$. Combining this with $(2.15)$, we have

$$
w_{t}+\nabla_{\bar{\alpha}} w V_{\alpha}+\nabla_{\alpha} w V_{\bar{\alpha}}+u_{\alpha \bar{\beta}} V_{\bar{\alpha}} V_{\beta}+\frac{w}{t} \geq 0 .
$$

Choosing $V_{\alpha}=-\frac{\nabla_{\alpha} w}{w}$ we conclude that (2.14) is true.

The last assertion follows from Proposition 2.2 immediately. 
Remark 2.1. If $u_{0}(x)$ is a solution to the Poincaré-Lelong equation $\sqrt{-1} \partial \bar{\partial} u_{0}=$ $\operatorname{Ric}(x, 0)$, by Theorem 1.3 of $[\mathrm{N}-\mathrm{T}]$ we know that we have a solution $u(x, t)$ to (2.1) in this case with $\sqrt{-1} \partial \bar{\partial} u(x, t)=\operatorname{Ric}(x, t)$. Then (2.14) in Theorem 2.1 is nothing but the differential LYH inequality of Cao on the scalar curvature since $w(x, t)=R(x, t)$.

Next we shall prove a Li-Yau type differential inequality for the positive plurisubharmonic solution of (2.1). The result will not be needed in the next section.

THEOREM 2.2. Let $u(x, t)$ be a positive solution to (2.1) such that $u(x, t)$ is plurisubharmonic for all $t$. Then we have the following differential inequality:

$$
\frac{u_{t}}{u}-\frac{|\nabla u|^{2}}{u^{2}}+\frac{m}{t} \geq 0
$$

Proof. As in Li-Yau [L-Y], we let $v=\log u$. Then

$$
\Delta v-v_{t}=-|\nabla v|^{2} .
$$

Let

$$
G(x, t)=t\left(|\nabla v|^{2}-\eta v_{t}\right)
$$

where $\eta>1$ is a constant. Direct calculation shows that in normal coordinates at a point:

$$
\begin{aligned}
\Delta|\nabla v|^{2}= & R_{\bar{\alpha} \beta} v_{\alpha} v_{\bar{\beta}}+v_{\alpha \gamma} v_{\bar{\alpha} \bar{\gamma}}+v_{\alpha \bar{\gamma}} v_{\bar{\alpha} \gamma}+(\Delta v)_{\alpha} v_{\bar{\alpha}}+v_{\alpha}(\Delta v)_{\bar{\alpha}} \\
& \frac{\partial}{\partial t}|\nabla v|^{2}=R_{\bar{\alpha} \beta} v_{\alpha} v_{\bar{\beta}}+\left(v_{t}\right)_{\alpha} v_{\bar{\alpha}}+v_{\alpha}\left(v_{t}\right)_{\bar{\alpha}}
\end{aligned}
$$

and

$$
v_{t t}-\Delta\left(v_{t}\right)=R_{\bar{\alpha} \beta} v_{\alpha \bar{\beta}}+R_{\bar{\alpha} \beta} v_{\alpha} v_{\bar{\beta}}+\left(v_{t}\right)_{\alpha} v_{\bar{\alpha}}+v_{\alpha}\left(v_{t}\right)_{\bar{\alpha}} .
$$

Combining (2.16)-(2.19) we have that

$$
\begin{aligned}
\left(\Delta-\frac{\partial}{\partial t}\right)\left(|\nabla v|^{2}-\eta v_{t}\right)= & v_{\alpha \gamma} v_{\bar{\alpha} \bar{\gamma}}+v_{\alpha \bar{\gamma}} v_{\bar{\alpha} \gamma}-\left(|\nabla v|^{2}-\eta v_{t}\right)_{\alpha} v_{\bar{\alpha}} \\
& -v_{\alpha}\left(|\nabla v|^{2}-\eta v_{t}\right)_{\bar{\alpha}}+\eta R_{\bar{\alpha} \beta}\left(v_{\alpha \bar{\beta}}+v_{\alpha} v_{\bar{\beta}}\right) .
\end{aligned}
$$

Using the fact that

$$
R_{\bar{\alpha} \beta}\left(v_{\alpha \bar{\beta}}+v_{\alpha} v_{\bar{\beta}}\right)=\frac{1}{u} R_{\bar{\alpha} \beta} u_{\alpha \bar{\beta}} \geq 0
$$


we then have

$$
\begin{aligned}
\left(\Delta-\frac{\partial}{\partial t}\right) G & \geq t v_{\alpha \bar{\gamma}} v_{\bar{\alpha} \gamma}-2\langle\nabla G, \nabla v\rangle-\frac{G}{t} \\
& \geq \frac{t}{m}(\Delta v)^{2}-2\langle\nabla G, \nabla v\rangle-\frac{G}{t} \\
& =\frac{t}{m}\left(|\nabla v|^{2}-v_{t}\right)^{2}-2\langle\nabla G, \nabla v\rangle-\frac{G}{t} .
\end{aligned}
$$

Once we have (2.20), we can use the cut-off function argument as in [L-Y] to carry the interior estimates. For the sake of the completeness we include the argument here. Let $\psi(s)$ be a cut-off function such that $0 \leq \psi \leq 1, \psi(s) \equiv 1$ for $s \in[0,1]$ and $\psi(s) \equiv 0$ for $s \geq 2$. We also require that

$$
\psi^{\prime} \leq 0, \quad \psi^{\prime \prime} \geq-C_{1} \quad \text { and } \quad \frac{\left|\psi^{\prime}\right|^{2}}{\psi} \leq C_{1}
$$

for some positive constant $C_{1}$. Now we let $\phi(x)=\psi\left(r_{t}(x) / R\right)$. Let $\Phi=\phi G$. Suppose $\Phi$ attains a positive maximum at $\left(x_{0}, t_{0}\right)$. Then we have at $\left(x_{0}, t_{0}\right)$ :

$$
0 \geq\left(\Delta-\frac{\partial}{\partial t}\right) \Phi \quad \text { and } \quad \nabla \Phi=0
$$

Note that $\phi$ may not be smooth at $x_{0}$ in the space variable, but we can always use the trick of Calabi as in [L-Y]. $\phi$ may not be smooth in the $t$ variable at $t_{0}$, but we can use the difference quotient so that the final result of the following computations is correct. Hence the above differential inequality together with (2.20) implies that at $\left(x_{0}, t_{0}\right)$

$$
\begin{aligned}
0 \geq & t_{0} \phi\left(\Delta-\frac{\partial}{\partial t}\right) \Phi \\
\geq & \frac{1}{m}\left(t_{0} \phi|\nabla v|^{2}-t_{0} \phi v_{t}\right)^{2}-2 G t_{0} \frac{|\nabla \phi|^{2}}{\phi}+t\left(\Delta \phi-\frac{\partial}{\partial t} \phi\right) G \\
& -G \phi^{2}+2\langle\nabla \phi, \nabla v\rangle G \phi t_{0} \\
\geq & \frac{1}{m}\left(t_{0} \phi|\nabla v|^{2}-t_{0} \phi v_{t}\right)^{2}-G\left[2 t_{0} \frac{|\nabla \phi|^{2}}{\phi}-t_{0}\left(\Delta \phi-\frac{\partial}{\partial t} \phi\right)+1\right] \\
& -2 \frac{|\nabla \phi|}{\phi^{1 / 2}} G\left(|\nabla v| \phi^{1 / 2} t_{0}^{1 / 2}\right) t_{0}^{1 / 2} .
\end{aligned}
$$

Using (2.21) we have that

$$
\frac{|\nabla \phi|^{2}}{\phi} \leq \frac{C_{2}}{R^{2}} \quad \text { and } \quad-\Delta \phi \geq \frac{C_{2}}{R^{2}}
$$


Also Theorem 17.2 of [H4] implies that

$$
\left|\frac{\partial}{\partial t} \phi\right| \leq \frac{C_{2}}{R}
$$

Here $C_{2}$ is a constant dependent of $C_{1}, m$ and the upper bound of $|R m|(x, t)$. Combining (2.22)-(2.24) we have, at the maximum of $\Phi$ over $M \times[0, T]$, that

$$
0 \geq \frac{1}{m}(y-z)^{2}-\frac{C_{2}}{R}(y-\eta z) y^{1 / 2} t_{0}^{1 / 2}-(y-\eta z)\left(\frac{C_{2} t_{0}}{R^{2}}+\frac{C_{2} t_{0}}{R}+1\right) .
$$

Here $y=t_{0} \phi|\nabla v|^{2}\left(x_{0}, t_{0}\right), z=t_{0} \phi v_{t}\left(x_{0}, t_{0}\right)$. Using the trick of [L-Y], we write

$$
(y-z)^{2}=\frac{1}{\eta^{2}}(y-\eta z)^{2}+2 \frac{\eta-1}{\eta}(y-\eta z) y+\left(\frac{\eta-1}{\eta}\right)^{2} y^{2} .
$$

Using the $a x^{2}+b x \geq-\frac{b^{2}}{4 a}$, for $R \gg 1$ we have that

$$
0 \geq \frac{1}{m \eta^{2}}(y-\eta z)^{2}-(y-\eta z)\left(\frac{C_{3} t_{0}}{R}+1\right)
$$

for some constant $C_{3}$ independent of $R$. From which we have that

$$
\sup _{B_{0}(o, R) \times[0, T]} t\left(|\nabla v|^{2}-\eta v_{t}\right) \leq m \eta^{2}\left(1+\frac{C\left(m, \eta, T, \sup _{M \times[0, T]}|R m|\right)}{R}\right) .
$$

Here we have used the fact that $g(t)$ is nonincreasing so that $B_{t}(o, R) \supset B_{0}(o, R)$. Letting $R \rightarrow \infty$ and then $\eta \rightarrow 1$ we have (2.15).

3. Liouville properties of plurisubharmonic functions. In this section, we shall discuss Liouville properties of plurisubharmonic functions using the LYH type inequality in $\S 1$ and the results of $\S 2$. In this section, we always assume that $\left(M, g_{\alpha \bar{\beta}}(x)\right)$ is a complete noncompact Kähler manifold with bounded nonnegative holomorphic bisectional curvature. We also assume that for all $x \in M$ and $r>0$, $k(x, r) \leq \epsilon(r)$ for some nonincreasing function $\epsilon(r)$ with $\lim _{r \rightarrow \infty} \epsilon(r)=0$, where

$$
k(x, r)=f_{B_{0}(x, r)} \mathcal{R}_{0} d V_{0}
$$

and $\mathcal{R}_{0}$ is the scalar curvature of $M, g_{\alpha \bar{\beta}}(x)$. By [N-T], we know that (1.1) has a solution $g_{\alpha \bar{\beta}}(x, t)$ on $M \times[0, \infty)$ such that for any $0<T<\infty, g_{\alpha \bar{\beta}}$ satisfies (i)-(iv) in $\S 1$. 
Define

$$
F(x, t)=\log \left(\frac{\operatorname{det}\left(g_{\alpha \bar{\beta}}(x, t)\right)}{\operatorname{det}\left(g_{\alpha \bar{\beta}}(x, 0)\right)}\right)
$$

To illustrate the idea of the proof to a more general result, let us begin with the following particular case. In this case, what we need is to assume that (1.1) has long time solution $g_{\alpha \bar{\beta}}$ so that for any $T<\infty$, conditions (i)-(iv) in $\S 1$ are satisfied by $g_{\alpha \bar{\beta}}$ on $M \times[0, T]$.

THEOREM 3.1. With the above assumptions, suppose $u_{0}$ is a plurisubharmonic function such that (i) $u$ is bounded; and (ii) $\widetilde{\Delta} u_{0}(x) \leq \exp \left(a\left(1+r_{0}(x)\right)\right.$ for some constant $a>0$. Then $u_{0}$ must be constant.

Proof. Let $\widetilde{\Delta} u_{0}=f$, then $f \geq 0$. Since $u_{0}$ is bounded, by [N-S-T1, Corollary 2.1] we have

$$
\int_{0}^{\infty} s\left(f_{B_{0}(x, s)} f d V_{0}\right) d s \leq C_{1}
$$

for some constant $C_{1}$ independent of $x$. By [N-S-T1, Corollary 1.2], we know that

$$
\sup _{M}\left|\widetilde{\nabla} u_{0}\right| \leq C_{2}
$$

By Proposition 2.1, there is a unique solution $u(x, t)$ with initial data $u_{0}$. Moreover, by Proposition 2.1 and the maximum principle in [N-T, Theorem 1.2], we conclude that $u$ is uniformly bounded.

Since $\widetilde{\Delta} u_{0}(x) \leq \exp \left(a\left(1+r_{0}(x)\right)\right.$, by Theorem 2.1(i) we conclude that $u(x, t)$ is plurisubharmonic for all $t>0$. Moreover, suppose $u_{0}$ is not harmonic, then by Theorem 2.1(ii) $w=u_{t}>0$ for $t>0$ and $t w$ is nondecreasing in $t$. Hence

$$
\begin{aligned}
u(x, t)-u_{0}(x) & =\int_{0}^{t} w(x, s) d s \\
& \geq w(x, 1) \int_{1}^{t} \frac{1}{s} d s \\
& =w(x, 1) \log t
\end{aligned}
$$

Since $w(x, 1)>0$, let $t \rightarrow \infty$, the above inequality contradicts the fact that $u$ is uniformly bounded. Hence $u_{0}$ must be harmonic and is constant by [Y].

Next we shall generalize Theorem 3.1 by relaxing the condition that $u_{0}$ is bounded. In the following, we always assume that $u_{0}$ is a plurisubharmonic func- 
tion on $M$ such that there exists a constant $a>0$ such that

$$
\left\{\begin{array}{l}
\left|\widetilde{\nabla} u_{0}(x)\right| \leq a, \\
\widetilde{\Delta} u_{0}(x) \leq \exp \left(a\left(1+r_{0}(x)\right)\right.
\end{array}\right.
$$

for all $x \in M$. Note that in the proof of Theorem 3.1, we know that if $u_{0}$ is bounded, then $u_{0}$ will satisfy the first inequality of (3.3).

Because of (3.3), let $u$ be the solution of (2.1) with initial data $u_{0}$ constructed in Proposition 2.1. By Proposition 2.2, $u$ is plurisubharmonic for all $t \geq 0$. Let $v(x, t)=u(x, t)-u_{0}(x)$. Also, let $\mathfrak{m}(t)=\inf _{x \in M} F(x, t)$. Then $\mathfrak{m}(t) \leq 0$, nonincreasing, and is finite for fixed $t$ by properties (ii) and (iv) in $\S 1$ and the fact that $F(x, t)=-\int_{0}^{t} \mathcal{R}\left(\S, \int\right) d s$, where $\mathcal{R}\left(\S, \int\right)$ is the scalar curvature at time $s$.

LEMMA 3.1. With the above assumptions and notations, we have

$$
\widetilde{\Delta} v-e^{F} v_{t} \geq-\widetilde{\Delta} u_{0}
$$

Proof. As in [Shi, p. 156], using the fact that $g_{\alpha \bar{\beta}}$ is nonincreasing, we have

$$
\widetilde{\Delta} u \geq e^{F} \Delta u=e^{F} u_{t}=e^{F} v_{t}
$$

Hence

$$
\widetilde{\Delta} v=\widetilde{\Delta} u-\widetilde{\Delta} u_{0} \geq e^{F} v_{t}-\widetilde{\Delta} u_{0}
$$

The result follows.

LEMMA 3.2. With the same assumptions and notations as in Lemma 3.1, there is a constant $C$ such that for all $(x, t) \in M \times[0, \infty)$, we have

$$
0 \leq v(x, t) \leq C t^{\frac{1}{2}}(-\mathfrak{m}(2 t)+1)
$$

Proof. First note that $v(x, 0)=0$ and $v_{t}=u_{t}=\Delta u \geq 0$. Hence $v \geq 0$. We need a more refined estimate of (2.10). More precisely, the Bochner formula on $\left\|u_{\alpha \bar{\beta}}\right\|^{2}$ says that

$$
\left(\Delta-\frac{\partial}{\partial t}\right)\left\|u_{\alpha \bar{\beta}}\right\|^{2} \geq\left\|u_{\alpha \bar{\beta} \gamma}\right\|^{2}+\left\|u_{\alpha \bar{\beta} \bar{\gamma}}\right\|^{2}-\mathcal{R}(x, t)\left\|u_{\alpha \bar{\beta}}\right\|^{2} .
$$

Using the LYH type inequality of H.-D. Cao as in [N-T] we have that

$$
t \mathcal{R}(x, t) \leq-2 \mathfrak{m}(2 t)
$$


Combining them we have that

$$
\left(\Delta-\frac{\partial}{\partial t}\right)(1+t \Phi)^{\frac{1}{2}} \geq-(-\mathfrak{m}(2 t)+1) \Phi
$$

Here $\Phi=\left\|u_{\alpha \bar{\beta}}\right\|^{2}$. Now we can proceed as in the proof of Proposition 2.2 (iii) to conclude that

$$
\sup _{M \times[0, T]}\left((-\mathfrak{m}(2 T)+1)|\nabla u|^{2}+(1+t \Phi)^{\frac{1}{2}}\right) \leq a^{2}(-\mathfrak{m}(2 T)+1)+1,
$$

which then implies

$$
\left\|u_{\alpha \bar{\beta}}\right\|(x, t) \leq C_{1} t^{-\frac{1}{2}}(-\mathfrak{m}(2 t)+1)
$$

for some constant $C_{1}$ depending only on $m$ and $\sup _{M}\left|\widetilde{\nabla} u_{0}\right|$. Hence

$$
\begin{aligned}
v(x, T) & =\int_{0}^{T} v_{t}(x, t) d t \\
& =\int_{0}^{T} u_{t}(x, t) d t \\
& =\int_{0}^{T} \Delta u(x, t) d t \\
& \leq C_{1} \int_{0}^{T} t^{-\frac{1}{2}}(-\mathfrak{m}(2 t)+1) d t \\
& \leq C_{2} T^{\frac{1}{2}}(-\mathfrak{m}(2 T)+1)
\end{aligned}
$$

for some constant $C_{2}$ independent of $x$ and $t$. The proof of the lemma is completed.

Using the method of proof of Theorem 2.1 in $[\mathrm{N}-\mathrm{T}]$, we have:

THEOREM 3.2. Let $u_{0}$ be a plurisubharmonic function on $M$ satisfying (3.3). Suppose

$$
\limsup _{R \rightarrow \infty} \frac{\sup _{\partial B_{0}(o, R)} u_{0}}{\log t} \leq 0
$$

where $R^{2}=t^{\frac{3}{2}} e^{-\mathfrak{m}(2 t)}(-\mathfrak{m}(2 t)+1)$, then $u_{0}$ must be constant.

Remarks.

(a) It is easy to see that $R \rightarrow \infty$ if and only if $t \rightarrow \infty$.

(b) By [Sh2-3] and [N-T, Remark 2.2] if $\epsilon(r)$ at the beginning of this section satisfies $\epsilon(r) \leq C r^{-2}$ or more generally if $\int_{0}^{r} s \epsilon(s) d s \leq C \log (r+2)$, then $-\mathfrak{m}(t) \leq$ 
$C^{\prime} \log (t+1)$, and the assumption (3.6) can be replaced by

$$
\limsup _{x \rightarrow \infty} \frac{u_{0}(x)}{\log r_{0}(x)} \leq 0
$$

(c) Similarly, if $\epsilon(r) \leq r^{-\theta}$ for some $\theta>0$, then the assumption (3.6) can be replaced by

$$
\limsup _{x \rightarrow \infty} \frac{u_{0}(x)}{\log \log r_{0}(x)} \leq 0
$$

If $\int_{0}^{r} s \epsilon(s) d s \leq C r^{2} / \log (2+r)$, then the assumption (3.6) can be replaced by

$$
\limsup _{x \rightarrow \infty} \frac{u_{0}(x)}{\log \log \log r_{0}(x)} \leq 0
$$

and so on.

(d) It is easy to see that Theorem 3.1 is a particular case of Theorem 3.2.

Proof of Theorem 3.2. Let $u$ and $v$ as in Lemmas 3.1 and 3.2. Let $\left(x_{0}, T\right) \in$ $M \times(0, \infty)$. For any $R>0$, let $G_{R}$ be the positive Green's function with zero boundary value on $B_{0}\left(x_{0}, R\right)$ with respect to the initial metric. By (3.4)

$$
\begin{aligned}
& \int_{0}^{T} \int_{B_{0}\left(x_{0}, R\right)} G_{R}\left(x_{0}, y\right) \widetilde{\Delta} v(y, t) d V_{0} d t \\
& \geq-T \int_{B_{0}\left(x_{0}, R\right)} G_{R}\left(x_{0}, y\right) \widetilde{\Delta} u_{0}(y) d V_{0} \\
& \quad+\int_{0}^{T} \int_{B_{0}\left(x_{0}, R\right)} G_{R}\left(x_{0}, y\right) e^{F(y, t)} v_{t}(y, t) d V_{0} d t \\
& \geq-T \int_{B_{0}\left(x_{0}, R\right)} G_{R}\left(x_{0}, y\right) \widetilde{\Delta} u_{0}(y) d V_{0} \\
&+e^{\mathfrak{m}(T)} \int_{B_{0}\left(x_{0}, R\right)} G_{R}\left(x_{0}, y\right) v(y, T) d V_{0} \\
& \geq C_{1}(m)\left[-T\left(-u_{0}\left(x_{0}\right)+\sup _{B_{0}\left(x_{0}, R\right)} u_{0}\right)+e^{\mathfrak{m}(T)} R^{2} f_{B_{0}\left(x_{0}, \frac{R}{5}\right)} v(y, T) d V_{0}\right]
\end{aligned}
$$

for some positive constant $C_{1}$ depending only on $m$, where we have used Theorem 2.1 in [N-S-T1] and Lemma 2.2 in $[\mathrm{N}-\mathrm{T}]$ and the fact that $v \geq 0, F_{t} \leq 0$. On the other hand, by Green's formula and Lemma 3.2, we have that, for any $0<t<T$,

$$
\begin{aligned}
\int_{B_{0}\left(x_{0}, R\right)} G_{R}\left(x_{0}, y\right) \widetilde{\Delta} v(y) d V_{0} & =-v\left(x_{0}, t\right)-\int_{\partial B_{0}\left(x_{0}, R\right)} v \frac{\partial G_{R}}{\partial \nu} \\
& \leq C_{2} t^{\frac{1}{2}}(-\mathfrak{m}(2 t)+1)
\end{aligned}
$$


for some constant $C_{2}$ independent of $(x, t)$. By (3.4) and the fact that $v_{t} \geq 0$, we have $\widetilde{\Delta} v \geq-\widetilde{\Delta} u_{0}$. Since $v \geq 0$, by the generalized mean value inequality [N-T, Lemma 2.1], (3.7) and (3.8), we have

$$
\begin{aligned}
v\left(x_{0}, T\right) \leq & C_{3} f_{B_{0}\left(x_{0}, \frac{R}{5}\right)} v(y, T) d V_{0}+\int_{B_{0}\left(x_{0}, \frac{R}{5}\right)} G_{\frac{R}{5}}\left(x_{0}, y\right) \tilde{\Delta} u_{0}(y) d V_{0} \\
\leq C_{4} & {\left[R^{-2} T e^{-\mathfrak{m}(2 T)}\left(-u_{0}\left(x_{0}\right)+\sup _{B_{0}(o, 2 R)} u_{0}+T^{\frac{1}{2}}(-\mathfrak{m}(2 T)+1)\right)\right.} \\
& \left.\quad-u_{0}\left(x_{0}\right)+\sup _{B_{0}(o, 2 R)} u_{0}\right]
\end{aligned}
$$

if $R$ is large, for some constants $C_{3}$ and $C_{4}$ independent of $\left(x_{0}, T\right)$ and $R$. Let $R$ be such that $(2 R)^{2}=T^{\frac{3}{2}}(1+T) e^{-\mathfrak{m}(2 T)}(-\mathfrak{m}(2 T)+1)$, then by (3.6), we can conclude that

$$
\limsup _{t \rightarrow \infty} \frac{v\left(x_{0}, t\right)}{\log t}=0
$$

We claim that $u_{0}$ is harmonic. Suppose not, then as in the proof of Theorem 3.1, we have $u\left(x_{0}, t\right) \geq C \log t$ for some constant $C>0$ for all $t \geq 1$. This is impossible.

By the definition of $R$ in (3.6), it is easy to see that $\log R \geq \log t$ when $t$ is large. Hence (3.6) implies that

$$
\limsup _{R \rightarrow \infty} \frac{\sup _{\partial B_{0}(o, R)} u_{0}}{\log R}=0 .
$$

Since $u_{0}$ is harmonic, it must be constant by [C-Y].

Since one can solve the Poincaré-Lelong equation for a $(1,1)$ form on a complete noncompact manifold with nonnegative holomorphic bisectional curvature under rather weak assumptions on the $(1,1)$ form (see [N-S-T1]), one can apply Theorem 3.2 (or Theorem 3.1) to obtain results on the flatness of the holomorphic line bundles. As an example, we have the following:

COROLlaRY 3.1. Let $\left(M, g_{\alpha \bar{\beta}}(x)\right)$ be a complete nocompact Kähler manifold with bounded nonnegative holomorphic bisectional curvature satisfying the conditions in Theorem 3.1. Let $\left(L, \mathfrak{h}_{0}\right)$ be a holomorphic line bundle on $M$ with the Hermitian metric $\mathfrak{h}_{0}$. Suppose $\Omega\left(\mathfrak{h}_{0}\right) \geq 0$ and suppose its trace $\mathcal{S}_{0}=g^{\alpha \bar{\beta}}(x) \Omega_{\alpha \bar{\beta}}\left(\mathfrak{h}_{0}\right)(x)$ is bounded and

$$
\int_{0}^{\infty} s f_{B_{0}(x, s)} \mathcal{S}_{0}(y) d y d s \leq C
$$

for some constant $C>0$ for all $x \in M$. Then $\left(L, \mathfrak{h}_{0}\right)$ is flat. 
Proof. Using the fact that $\mathcal{S}_{0}$ is bounded and (3.10), one can find bounded function $u_{0}$ such that $\sqrt{-1} \partial \bar{\partial} u_{0}=\Omega\left(\mathfrak{h}_{0}\right)$ by [N-S-T1, Theorem 5.1]. Since $\Omega\left(\mathfrak{h}_{0}\right)$ is nonnegative, $u_{0}$ is plurisubharmonic. By Theorem 3.1, $u_{0}$ is constant and hence $\left(L, \mathfrak{h}_{0}\right)$ is flat.

DePARTMENT of MAThEMATiCs, University of CALifornia, LA Jolla, CA 92093

E-mail: lni@math.ucsd.edu

Department of Mathematics, The Chinese University of Hong Kong, Shatin, HONG KONG, CHINA

E-mail:1ftam@math.cuhk.edu.hk

\section{REFERENCES}

[A] B. Andrews, Harnack inequalities for evolving hypersurfaces, Math. Z. 217 (1994), 179-197.

[Co1] H.-D. Cao, On Harnack inequalities for the Kähler-Ricci flow, Invent. Math. 109 (1992), 247-263.

[Co2] Limits of solutions to the Kähler-Ricci flow, J. Differential Geom. 45 (1997), 257-272.

[C-Z] B. L. Chen and X. P. Zhu, On complete noncompact Kähler manifolds with positive bisectional curvature, preprint.

[C-T-Z] B. L. Chen, S. H. Tang and X. P. Zhu, A uniformization theorem of complete noncompact Kähler surfaces with positive bisectional curvature, preprint.

[C-Y] S. Y. Cheng and S.-T. Yau, Differential equations on Riemannian manifolds and their geometric applications, Comm. Pure Appl. Math. 28 (1975), 333-354.

[Cw1] B. Chow, On Harnack's inequality and entropy for the Gaussian curvature flow, Comm. Pure Appl. Math. 44 (1991), 469-483.

[Cw2] The Yamabe flow on locally conformally flat manifolds with positive Ricci curvature, Comm. Pure Appl. Math. 45 (1992), 1003-1014.

[Cw3] Licci flow and Einstein metrics in low dimensions, Surv. Differ. Geom., vol. 6, Int. Press, Boston, MA, 1999, pp. 187-220.

[C-H] B. Chow and R. Hamilton, Constrained and linear Harnack inequalities for parabolic equations, Invent. Math. 129 (1997), 213-238.

[H1] R. S. Hamilton, Three-manifolds with positive Ricci curvature, J. Differential Geom. 17 (1982), 255-306.

[H2] $\quad$ Four-manifolds with positive curvature operator, J. Differential Geom. 24 (1986), 153-179.

[H3] The Harnack estimate for the Ricci flow, J. Differential Geom. 37 (1993), 225-243.

[H4] Formation of singularities in the Ricci flow, Surv. Differ. Geom., vol. 2, Int. Press, Boston, MA, 1995, pp. 7-136.

[L-Y] P. Li and S.-T. Yau, On the parabolic kernel of the Schrödinger operator, Acta Math. 156 (1986), 139-168.

[M1] N. Mok, An embedding theorem of complete Kähler manifolds of positive bisectional curvature onto affine algebraic varieties, Bull. Soc. Math. France 112 (1984), 197-250.

[M2] The uniformization theorem for compact Kähler manifolds of nonnegative holomorphic bisectional curvature, J. Differential Geom. 27 (1988), 179-214.

[M-S-Y] N. Mok, Y.-T. Siu and S.-T. Yau, The Poincaré-Lelong equation on complete Kähler manifolds, Compositio Math. 44 (1981), 183-218.

[N-S-T1] L. Ni, Y.-G. Shi and L.-F. Tam, Poisson equation, Poincaré-Lelong equation and curvature decay on complete Kähler manifolds, J. Differential Geom. 57 (2001), 339-388. 
[N-S-T2] — Ricci flatness of asymptotically locally flat Euclidean metrics, Trans. Amer. Math. Soc. (to appear).

[N-T] L. Ni and L.-F. Tam, Kähler-Ricci flow and Poinaré-Lelong equation, Comm. Anal. Geom (to appear).

[Sh1] W. X. Shi, Deforming the metric on complete Riemannian manifolds, J. Differential Geom. 30 (1989), 223-301.

[Sh2] Ricci deformation of metric on complete noncompact Kähler manifolds, Ph.D. thesis, Harvard University, 1990.

[Sh3] $\longrightarrow$ Ricci flow and the uniformization on complete noncompact Kähler manifolds, J. Differential Geom. 45 (1997), 94-220.

[Y] S.-T. Yau, Harmonic functions on complete Riemannian manifolds, Comm. Pure Appl. Math. 28 (1975), 201-228. 